PREPARED FOR THE U.S. DEPARTMENT OF ENERGY, UNDER CONTRACT DE-AC02-76CH03073

PPPL-3936

PPPL-3936

UC-70

Observation of SOL Current Correlated with MHD Activity

in NBI Heated DIII-D Tokamak Discharges

by

H. Takahashi, E.D. Fredrickson, M.J. Schaffer, M.E. Austin, T.E. Evans, L.L. Lao, and J.G. Watkins

March 2004
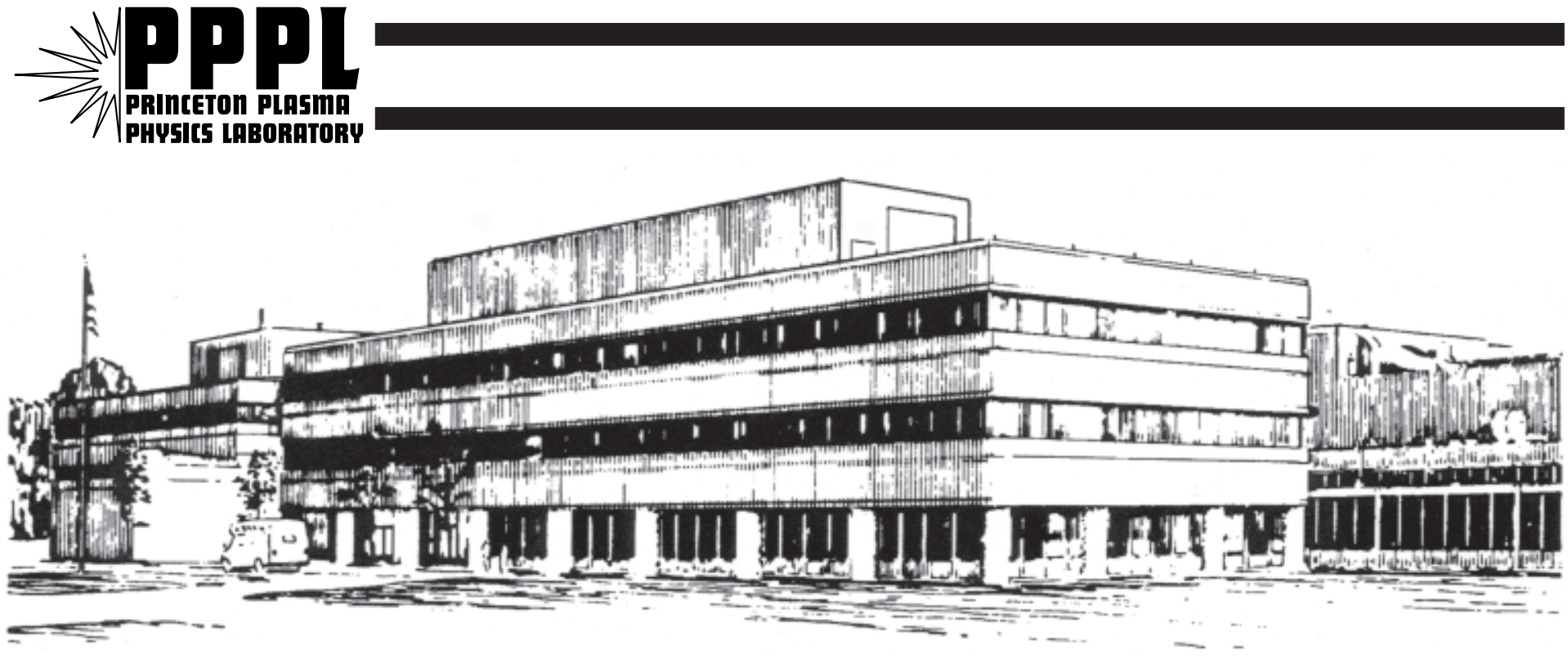

PRINCETON PLASMA PHYSICS LABORATORY PRINCETON UNIVERSITY, PRINCETON, NEW JERSEY 


\section{PPPL Reports Disclaimer}

This report was prepared as an account of work sponsored by an agency of the United States Government. Neither the United States Government nor any agency thereof, nor any of their employees, makes any warranty, express or implied, or assumes any legal liability or responsibility for the accuracy, completeness, or usefulness of any information, apparatus, product, or process disclosed, or represents that its use would not infringe privately owned rights. Reference herein to any specific commercial product, process, or service by trade name, trademark, manufacturer, or otherwise, does not necessarily constitute or imply its endorsement, recommendation, or favoring by the United States Government or any agency thereof. The views and opinions of authors expressed herein do not necessarily state or reflect those of the United States Government or any agency thereof.

\section{Availability}

This report is posted on the U.S. Department of Energy's Princeton Plasma Physics Laboratory Publications and Reports web site in Fiscal Year 2004. The home page for PPPL Reports and Publications is: http://www.pppl.gov/pub_report/

DOE and DOE Contractors can obtain copies of this report from:

U.S. Department of Energy

Office of Scientific and Technical Information

DOE Technical Information Services (DTIS)

P.O. Box 62

Oak Ridge, TN 37831

Telephone: (865) 576-8401

Fax: (865) 576-5728

Email: reports@adonis.osti.gov

This report is available to the general public from:

National Technical Information Service

U.S. Department of Commerce

5285 Port Royal Road

Springfield, VA 22161

Telephone: $1-800-553-6847$ or

(703) $605-6000$

Fax: (703) 321-8547

Internet: http://www.ntis.gov/ordering.htm 


\title{
Observation of SOL current correlated with MHD activity in NBI heated DIII-D tokamak discharges
}

\author{
H. Takahashi ${ }^{1}$, E. D. Fredrickson ${ }^{1}$, M.J. Schaffer ${ }^{2}$, M.E. Austin ${ }^{3}$, T.E. Evans ${ }^{2}$, L.L. Lao ${ }^{2}$, \\ J.G. Watkins ${ }^{4}$ \\ ${ }^{1)}$ Princeton Plasma Physics Laboratory, Princeton University, Princeton, NJ, USA \\ ${ }^{2)}$ General Atomics, San Diego, CA, USA \\ ${ }^{3)}$ University of Texas, Austin, TX, USA \\ 4) Sandia National Laboratory, Albuquerque, NM, USA
}

\begin{abstract}
This work investigates the potential roles played by the scrape-off-layer current (SOLC) in MHD activity of tokamak plasmas, including effects on stability. SOLCs are found during MHD activity that are: (1) slowly growing after a mode-locking-like event, (2) oscillating in the several $\mathrm{kHz}$ range and phase-locked with magnetic and electron temperature oscillations, (3) rapidly growing with a sub-ms time scale during a thermal collapse and a current quench, and (4) spiky in temporal behavior and correlated with spiky features in $\mathrm{D}_{\square}$ signals commonly identified with the edge localized mode (ELM). These SOLCs are found to be an integral part of the MHD activity, with a propensity to flow in a toroidally non-axisymmetric pattern and with magnitude potentially large enough to play a role in the MHD stability. Candidate mechanisms that can drive these SOLCs are identified: (a) toroidally non-axisymmetric thermoelectric potential, (b) electromotive force (EMF) from MHD activity, and (c) flux swing, both toroidal and poloidal, of the plasma column. An effect is found, stemming from the shear in the field line pitch angle, that mitigates the efficacy of a toroially non-axisymmetric SOLC to generate a toroially nonaxisymmetric error field. Other potential magnetic consequences of the SOLC are identified: (i) its error field can introduce complications in feedback control schemes for stabilizing MHD activity, and (ii) its toroidally non-axisymmetric field can be falsely identified as an axisymmetric field by the tokamak control logic and in equilibrium reconstruction. The radial profile of a SOLC observed during a quiescent discharge period is determined, and found to possess polarity reversals as a function of radial distance.
\end{abstract}

\section{Introduction}

Currents have been observed to flow in tokamaks in the space between the plasma and the vacuum vessel, including the scrape-off layer (SOL) of the plasma. These are extraneous to the current that sustains magnetic confinement configuration of the plasma. Driving mechanisms for them vary, both demonstrated and presumed. This work investigates the possibility that these currents may play a role in MHD activity of tokamak plasmas, including effects on stability.

Extensive work has been performed to characterize the SOL of poloidally diverted tokamak discharges. The majority of this work has concerned the flux of heat and particles between the plasma and divertors. But as a result of these investigations, it has become known that electrical currents also flow in the SOL. Scrape-off layer currents (SOLCs) are believed to flow for the most part along open field lines in the SOL, and close their circuits through the tokamak structure. SOLCs were first observed in JET [1 $\square 3]$, and have since been found in many other tokamaks, including DIII-D [49], JT-60U [10, 11], JFT2M [12], and TCV [13].

The origin of the SOLC is not yet firmly established, and is the subject of continuing research. But among possible driving mechanisms that can be operative in steady quiescent discharges, a leading candidate is the thermoelectric potential [14 $\square 16]$ in which a temperature difference between the two ends of an 
open field line drives current along it. The pressure difference may also play a similar role $[2,10]$. The outward magnetic helicity transport [17] and Pfirsch-Schlüter effect in the SOL [3] have been proposed. The loop voltage and bootstrap effects have also been examined [5]. SOLCs have also been observed to flow during MHD activity. They have been seen during edge localized modes (ELMs) in many devices, including JET [1], DIIID [5】9], JFT2M [12] and TCV [13]. Toroidally non-axisymmetric and bi-polar variation of the SOLC during the ELM has been reported [6־9].

Large currents (significant fractions of the plasma current) are observed to flow in poloidally diverted tokamak discharges in the "vacuum" region between the plasma and the tokamak structure as a consequence of the loss of control of the vertical plasma position. These "halo" currents are driven first by a poloidal flux change caused by a largescale plasma motion (comparable to the plasma minor radius), and then by a toroidal flux swing associated with the contraction of the plasma cross section, mainly as a result of the plasma coming into contact with the tokamak structure and ending in a disruption. This sequence of events was first observed in JET [18], and has since become commonly known as the vertical displacement episode or event (VDE) [19]. The phenomenon has been extensively investigated in many other tokamaks [20-27]. The current during the VDE was found to evolve on the time scale of the plasma geometry changes, typically a few tens of $\mathrm{ms}$ in large tokamaks. The toroidally nonaxisymmetric nature of the current has been documented in many of these investigations. The VDE is not addressed in the present work, but the current driving mechanisms identified in its investigations are of interest.

Actively driving currents with an external power supply in the region between the plasma and tokamak structure has also been proposed [28 $\square 31]$ and demonstrated $[27,32]$ as a means of affecting the MHD stability and other discharge characteristics.

Investigation of the SOLC in the present work as a potential element in the MHD stability physics is new. A commonly employed model for stability analysis consists of three components, the plasma, the vessel wall and a "vacuum" region between them. The vessel wall is a passive element that reacts to MHD activity in the plasma through image currents that mirror, to lowest order, the geometrical structure of the MHD activity. Such reactive currents may be inherently stabilizing. The image current must nevertheless be included in MHD stability analysis, e.g., of resistive wall modes (RWMs), even though the current is stabilizing, because its inclusion dramatically affects the outcome of the analysis. The SOLC introduces an additional element, intervening between the plasma and the vessel wall, which may be affected, not only by MHD activity inside the plasma, but also by the field line topology in the SOL and the tokamak structural geometry. The SOLC, which can be driven through mechanisms independent of MHD activity, possesses the potential to be a destabilizing influence. The new element will need to be included in stability analysis, if the magnetic field it produces is large enough, be it stabilizing or destabilizing - a question yet to be answered.

Among candidate mechanisms for driving the SOLC during MHD activity are the electromotive force (EMF) of the MHD activity, and flux swing, both toroidal and poloidal, of the plasma column. The flux swing of the plasma column is a current driving mechanism already identified in the VDE, but may also be operative without large-scale motion or contraction of the plasma column, as the loss of thermal energy and changes in the plasma current also produce toroidal and poloidal flux swings, respectively. The SOLC during MHD activity is both smaller (by an order of magnitude), and evolves faster (by up to two orders of magnitude), in comparison with the halo current during the VDE. Mechanisms that can drive current in quiescent discharges may also be at work during MHD activity, perhaps in an altered or intensified form. The propensity for the SOLC to flow in a toroidally non-axisymmetric pattern, which is experimentally demonstrated, though not well understood, can result in generation of nonaxisymmetric field - a form of error field - even though the underlying driving mechanisms may be inherently axisymmetric. The SOLC may thus enter the MHD stability physics in the role of a 
"flux converter," generating helical flux at the expense of an axisymmetric one.

The error field generated by the SOLC comes and goes as the conditions vary. The SOLC is thus a dynamic source of error field. The SOLC may also have "magnetic consequences" beyond its potential influence on the MHD stability. The spatial structure of the dynamically varying error field generated by the SOLC, which may differ from that of MHD modes inside the plasma because the SOLC path is determined by the topology of field lines in the SOL, adds complications to a feedback stabilization scheme wherein the modes' spatial structure is detected and countered with fields generated by external coils, as in RWM feedback stabilization methods. A toroidally nonaxisymmetric error field detected by toroidally discrete magnetic sensors (e.g., Mirnov coils and saddle loops) may be falsely interpreted as an axisymmetric field by the tokamak control logic and in equilibrium reconstruction analysis.

The physical proximity of the SOLC to the plasma, particularly its edge regions, enhances the SOLC's magnetic consequences, both positive and negative, for a given amount of current, in comparison with currents in coils that are far from the plasma, particularly so in large devices such as ITER and future reactors. This opens the possibility of taking advantage of actively driven SOLCs as a means of controlling MHD activity. It is an objective of this work to better understand the nature of the SOLC to assess the feasibility of such schemes.

The experimental environment is described in Sec. 2. Operating regimes of DIII-D are briefly discussed. Pertinent diagnostics are described, including those for SOLC measurement and standard tokamak diagnostics. In Sec. 3 the range of the SOLC observed in DIII-D is described, including the advanced tokamak (AT) regime wherein magnetic phenomena, such as the RWM, neoclassical tearing mode (NTM), ELM, and locked mode (LM) may manifest themselves. Magnetic consequences are discussed in Sec. 4. Characteristics of the SOLC are listed that either enhance or diminish its magnetic consequences, including the toroidal non-axisymmetry, current path topology, "phase mixing," and radial profile.
A line current model is used to explain the phase mixing effect. Observations of toroidally nonaxisymmetric SOLCs are described in Sec. 5, which are correlated with three classes of MHD activity: a slow LM-like process, traveling oscillating magnetic perturbations, and a fast thermal collapse (loss of a significant amount of thermal energy, especially that of electrons, on a ms time scale) followed by a partial current quench (sudden change in the plasma current following a thermal collapse). A radial profile of the SOLC is determined using a "swept strike point method" in Sec. 6. Conclusions are drawn in Sec. 7.

Discussion is given in Sec. 8.

\section{Experimental Environment}

\subsection{Operating Regimes}

Measurement of the SOLC is performed in poloidally diverted, neutral beam injection (NBI) heated, H-mode discharges in the DIII-D tokamak [33]. The device's typical operating parameters are: plasma major radius, $\mathrm{R} \square 1.7 \mathrm{~m}$, plasma minor radius, a $\square 0.6 \mathrm{~m}$, toroidal field, $\mathrm{B}_{\mathrm{t}} \square 2.14 \mathrm{~T}$, at $\mathrm{R}=$ $1.7 \mathrm{~m}$, plasma current, $\mathrm{I}_{\mathrm{p}} \square 2 \mathrm{MA}$, plasma elongation, $\square \sim 1.8$. Its heating systems include NBI up to $16 \mathrm{MW}$.

In the DIII-D convention, both the $\mathrm{B}_{\mathrm{t}}$ and $\mathrm{I}_{\mathrm{p}}$ are defined to be positive in a counter-clock-wise direction when viewed from above. A combination of a positive $\mathrm{I}_{\mathrm{p}}$ and a negative $\mathrm{B}_{t}$ is the "standard" operating configuration. The standard configuration has magnetic field lines with a lefthanded twist.

The SOLC has been measured in different magnetic configurations, including upper single null (USN), lower single null (LSN), and double null (DN) diverted configurations, and with different NBI directions, i.e., either co- or counterinjection (parallel or anti-parallel to the ohmic plasma current). The SOLC has been correlated to MHD activity. But little systematic effort to date has been made to correlate the observed differences in the SOLC behavior to variations in the discharge. 
For a discharge aimed at high performance, a 'ruleof-thumb,' or nominal operational value of normalized $\square$ (ratio of thermal to magnetic energy), $\square_{\mathrm{N}} \equiv \square /\left(\mathrm{I}_{\mathrm{p}} / \mathrm{aB}_{\mathrm{t}}\right)$, is sometimes quoted for the $\square$ limit of kink modes in the absence of a conducting wall ("no-wall" limit). The nominal no-wall limit is $\approx 4$ times the dimensionless internal inductance, $l_{i}$, during an $\mathrm{I}_{\mathrm{p}}$ flat-top period, and $\approx 2.4$ times $l_{i}$ for an $\mathrm{I}_{\mathrm{p}}$ ramp-up period for DIII-D discharges aimed at high performance [34]. In this article the purpose of comparing $\square_{\mathrm{N}}$ with a no-wall limit is simply to indicate that observed SOLCs may be relevant to an $\mathrm{AT}$, not as a precise delineation of discharge regimes.

\subsection{SOLC measurement}

Resistive-element current sensors fitted between protective graphite tiles and the tokamak structure are used to measure the SOLC in DIII-D. Langmuir probes embedded in tiles may also be used for a more localized measurement. The data reported in this article are from the current sensors.

The protective graphite tiles in the divertor regions are arranged in a pattern of concentric rings on the tokamak structure, i.e., either the vacuum vessel wall or the divertor baffle plates, which are supported from the vessel wall by thin metallic struts. Figures 1 and 2 explain the tile arrangement for the top and bottom divertor regions,

respectively, each through two views of a divertor, one placed inside the other as an inset.

The outside ring-shaped drawing in either Fig. 1 or 2 shows tile placement in a radius-angle (toroidal) plane when viewed from above. The ring-shaped drawing is a schematic representation of the radial location of a ring with respect to the others and toroidal angular locations of individual tiles within it, not to scale. The ring numbers are indicated (magenta). The outer angle scale (blue) shows the toroidal angle coordinate in the DIII-D convention, which runs in a clock-wise direction when viewed from above. The inner angle scale (green), running in a counter-clock-wise direction, is for a righthanded coordinate system, which is employed in the analysis of magnetic field. The ring-shaped drawing is shown in two parts, separated by a gap for clarity; the inner four rings, 9-12, each consisting of 48 tiles, with an individual tile spanning a toroidal angle of about $7.5 \mathrm{deg}$, and the outer two rings, 13 and 14, each consisting of 72 tiles, with an individual tile covering a toroidal angle of about $5 \mathrm{deg}$.

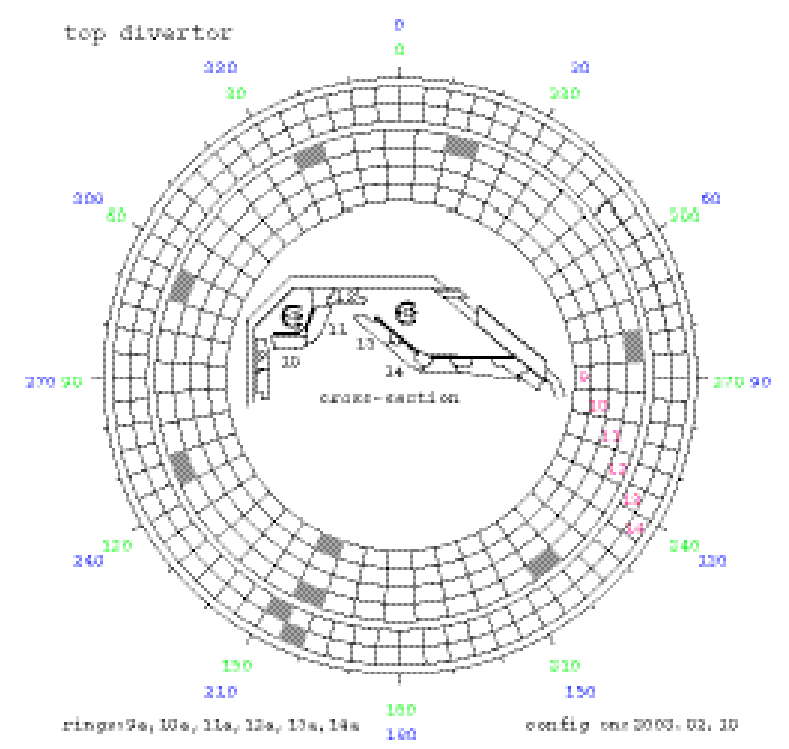

Fig. 1. Tile layout in the top divertor is shown; (outer ring-shaped drawing) schematic representation of tile locations in a radius-angle (toroidal) plane viewed from above. Tiles shown in gray shade are fitted with a current sensor; (inner inset drawing) poloidal crosssection, showing vessel wall, inboard and outboard pump chambers, and tiles.

The inset in the middle of the ring-shaped drawing in either Fig. 1 or 2 is a miniaturized engineering drawing of a divertor region, showing parts of the vacuum vessel wall, pumping chamber(s), and tiles, all in a poloidal cross-section. The ring numbers are also shown in this view (black).

A suffix letter, "A" or "B", will be added to the ring numbers for indicating either the top or bottom divertor, respectively. A set of a ring number and a toroidal angle designates an individual tile. For example, the tile, 12A200, is in ring \#12A in the top divertor and centered approximately at $200^{\circ}$ toroidal angle in the DIII-D convention. The tile ring \#10B, which lies at an angle of about $45^{\circ}$ in the inboard bottom corner of the vessel (see Fig. 2 inset), will also be referred to as the "slanted ring." 
Most of the tiles are in direct mechanical and electrical contact with the tokamak structure. A few selected tiles are electrically insulated from the structure, and then fitted with a resistive-element current sensor (about $2.3 \mathrm{~m} \square$ ), which measures current flowing between the tile and the structure. The current will be referred to as the "tile current." It is defined to be positive when flowing from the tile to the structure. The tiles shaded in gray in Figs. 1 and 2 are equipped with a tile current sensor. At most, about $10 \%$ of tiles in selected rings are monitored. The instrumented tile is electrically and mechanically separated from its neighbors by stand off gaps. The configuration of available instrumented tiles varied from time to time because of improvement, breakage, etc. The long-term stability of the sensitivity of the tile current sensors is estimated to be $\pm 20 \%$. SOLC data used in the present analysis are sampled at $200 \mathrm{kHz}$. (Figure 11 shows a line current model of an SOLC circuit.)

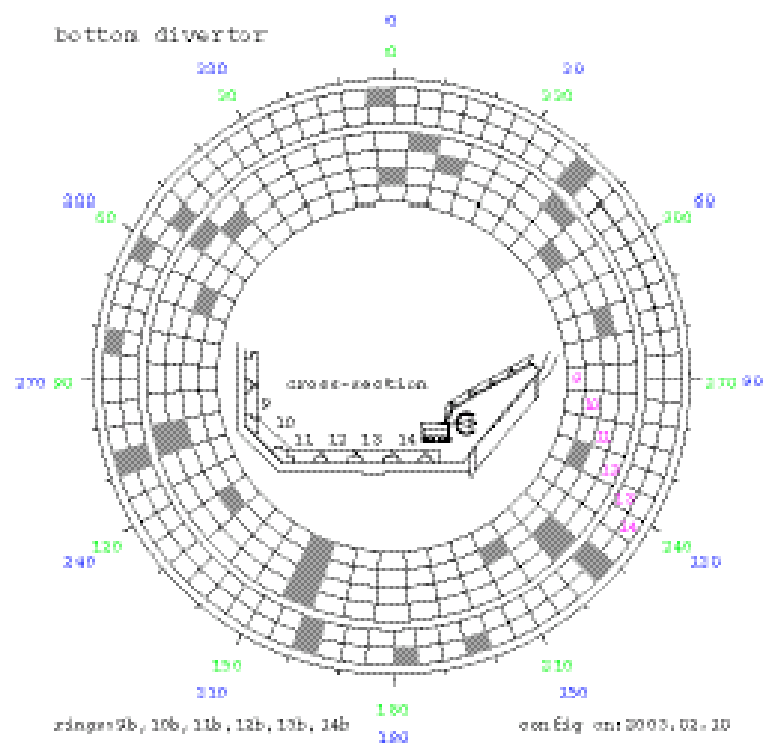

Fig. 2. Tile layout in the bottom divertor is shown; see caption to previous figure for explanation. The tile ring \#10B will be referred to also as the "slanted ring."

\subsection{Standard tokamak diagnostics}

A standard array of tokamak diagnostics available on DIII-D has been used in the data analysis. They include magnetic diagnostics [35], an electron cyclotron emission (ECE) diagnostic [36], a Thomson scattering (TS) diagnostic [37], a filter- scope diagnostic ( $\mathrm{D}_{\square}$ light) [38], charge exchange recombination (CER) spectroscopy [39], and a soft $\mathrm{X}$-ray (SXR) emission diagnostic [40]. A standard code used for calculating equilibrium in DIII-D is EFIT [41].

The magnetic diagnostics of interest here include Mirnov coils for measuring the poloidal field and saddle loops for the radial field. Mirnov coil data used in the present analysis are sampled either at $100 \mathrm{kHz}$ or $200 \mathrm{kHz}$, and saddle loop signals at 2 $\mathrm{kHz}$. The fast electron temperature measurements were made with an ECE radiometer which views a radial chord at the horizontal mid-plane with channels spaced approximately every $2 \mathrm{~cm}$ and with $2-3 \mathrm{~cm}$ spatial resolution. The signals are sampled at $2 \mathrm{kHz}$, covering the entire discharge, and $100 \mathrm{kHz}$ over part of the discharge. The TS diagnostic used here measures the electron temperature and density at discrete spatial points along a vertical line at $\mathrm{R}=194 \mathrm{~cm}$, and its measurement points fall both within the main plasma and the SOL, and are spaced about $1.3 \mathrm{~cm}$ apart in plasma edge regions and father apart in the interior of the plasma.

\section{Range of SOLC observed in DIII-D}

SOLCs observed in DIII-D are cataloged in this section without in-depth analysis to relate them to other measurements. The next section will examine some specific cases in greater detail in the context of other parameters commonly used for characterizing MHD activity.

\subsection{Driving Mechanisms of SOLC}

The physical mechanisms that drive the SOLC are not yet fully understood. But the thermoelectric potential is presently considered to be a leading candidate for SOLCs observed in quiescent discharges. There has been much less investigation of the origin of the SOLC during MHD activity, with the notable exceptions of the ELM and VDE. The thermoelectric potential, perhaps altered or intensified by the MHD activity itself through ejection of heat and plasma and fast particles into the SOL, is still a candidate mechanism even for SOLCs during MHD activity. 
Among other candidate mechanisms for driving the SOLC during MHD activity are the electromotive force (EMF) of non-axisymmetric MHD activity and flux swing, both toroidal and poloidal, of the plasma column. Both EMF and flux swing can produce an SOLC that is toroidally nonaxisymmetric. But reflecting the helical nature of MHD activity, its EMF produces a SOLC that is toroidally bi-polar - with the current direction reversing as a function of toroidal angle. But it is by itself incapable of generating a SOLC that is toroidally uni-polar - current flowing in the same direction everywhere. The opposite is true of a flux swing of the plasma column. It produces a unipolar SOLC, but is by itself incapable of generating a bi-polar SOLC. This difference in the characteristics will help to sort out candidate mechanisms operative under a given circumstance.

\subsection{Total Current Involved}

It is desirable to be able to qualitatively assess the potential impact of observed SOLCs by comparing their magnitude with other known currents, for example, the plasma current or coil currents used for MHD feedback control. This gives rise to the need for estimating how much current is involved. A "rule-of-thumb" estimate for the peak-to-peak "total current involved" may be provided by modeling the toroidal variation as a sinusoid: $\mathrm{N}\left(\mathrm{I}_{\max }-\mathrm{I}_{\min }\right) /(\mathrm{n} \square)$, where $\mathrm{N}$ is the number of tiles in a ring (48 or 72), $I_{\max }$ and $I_{\min }$ are the maximum and minimum values measured by a toroidal array of tile current sensors, and $\mathrm{n}>0$ is the toroidal harmonic number.

"Background" current observed in the absence of significant MHD activity, or in quiescent periods between occurrences of MHD activity such as ELMs, ranges from a few A to about 60 A per tile. As there is relatively little toroidal variation of these background currents measured by instrumented tiles, the estimated total current involved ranges up to $3 \mathrm{kA}$ (48 tiles times 60 A per tile). Oscillating SOLCs have peak-to-peak amplitudes ranging from a few $\mathrm{A}$ to $\sim 80 \mathrm{~A}$ through a single tile. The estimated total current involved is up to about $1.3 \mathrm{kA}$ for an $\mathrm{n}=1$ variation (48 tiles times 80 A per tile divided by 1 times $\square)$. The peak value of SOLC measured through a single tile is up to $200 \mathrm{~A}$ during an RWM, and 400 A during an ELM, measured at the top of the ELM spike. A thermal collapse involves a peak SOLC of up to 300 A per tile, and a partial current quench following a thermal collapse can have current in excess of $1.5 \mathrm{kA}$ per tile. The toroidally non-axisymmetric part varies from one case to another, but is often a substantial fraction of the peak value. The largest total current involved is thus estimated to be as much as a few kA for an RWM, ELM, or a thermal collapse, and up to a few tens of kA for a partial current quench. Total current involved will be estimated in each case discussed in this article.

To place the magnitude of these SOLCs in perspective, the in-vessel, one-turn, RWM feedback coil system ("I-coils") in DIII-D applies less than $5 \mathrm{kA}$ of current at a distance from the plasma many times that of the SOLC in an effort to control the MHD stability [42]. In comparison, the SOLC flows in the immediate proximity of the plasma edge. The SOLC thus appears to possess at least the potential to play a role in the MHD stability physics.

\subsection{SOLC in quiescent $\mathrm{H}$-mode discharge}

A class of discharges in an enhanced confinement regime known as the quiescent $\mathrm{H}$-mode (QHM) [43] offers an opportunity to examine the level of background SOLC in the absence of significant MHD activity, and thereby establish connection to past measurements in DIII-D and other tokamaks.

The QHM discharge does exhibit coherent and persistent magnetic oscillations in the range of several $\mathrm{kHz}$ to a few tens of $\mathrm{kHz}$ with multiple higher harmonics, which are known as the edge harmonic oscillations (EHOs) [43] or multiharmonic oscillations. But the amplitude of these magnetic oscillations is smaller than that of typical global MHD modes by up to two orders of magnitude.

Figure 3 summarizes the conditions of a QHM discharge in a compact pictorial form: the left panel describes the time variation of some discharge parameters of interest, and the right 
panel presents a poloidal flux surface plot, indicating the magnetic configuration of the discharge, together with numerical values of the toroidal field and plasma current at a single time point. This USN discharge, with $\mathrm{B}_{\mathrm{t}}<0$, has downward ion $\square \mathrm{B}$ drift, away from the $\mathrm{X}$-point, and with $\mathrm{I}_{\mathrm{p}}<0$, is heated with counter-injection. The discharge is initially in the QHM over the period, 1550-3800 ms, and exhibits EHOs. But it subsequently loses the QHM, developing small ELMs and fishbone-like bursts.

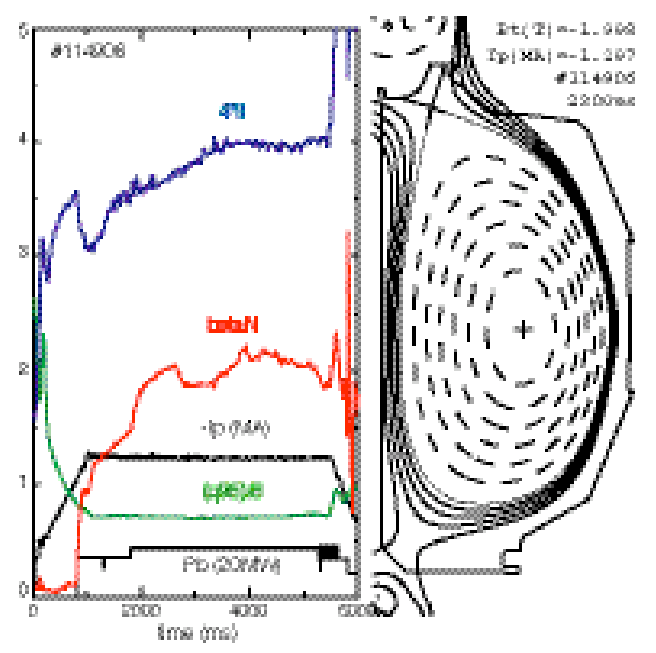

Fig. 3 Pictorial discharge summary; the left-hand panel shows $I_{p}$ in units of $M A, P_{b}$ in units of $20 M W, q_{95}$ divided by $6, \square_{N}$, and nominal no-wall limit (here, 4 li). The right-hand panel shows the plasma boundary and four exterior flux surfaces in the SOL in solid lines, and interior flux surfaces in dashed lines. The exterior surfaces pass through points 1, 2, 3, and $4 \mathrm{~cm}$ away from the plasma boundary in the outboard mid-plane in this figure and those to follow. This figure is for a USN QHM discharge with counter NBI.

Signals from two tile current sensors in the ring \#12A, one (ITLF12A295) at toroidal angle $295^{\circ}$ and another (ITLF12A340) at $340^{\circ}$, are shown in Fig. 4. The signal traces have spiky features and dark areas. The spiky features are mostly small ELMs (see next subsection), and the dark areas are produced by pile up of spiky features and oscillations that cannot be resolved on the time scale of the plots. The signals are nearly zero over the period, 2700-3200ms, when current flowing between the outboard SOL and the upper divertor region, just underneath the ring \#12A (see inset in Fig. 1), becomes interrupted because the gap between the separatrix and a protruding leading edge of the neighboring tile ring \#13A narrows down or closes completely. "Closed gap" and "open gap" configurations will be explained in more detail when the radial distribution of a SOLC is determined later (see Fig. 23 in Sec. 6.3).

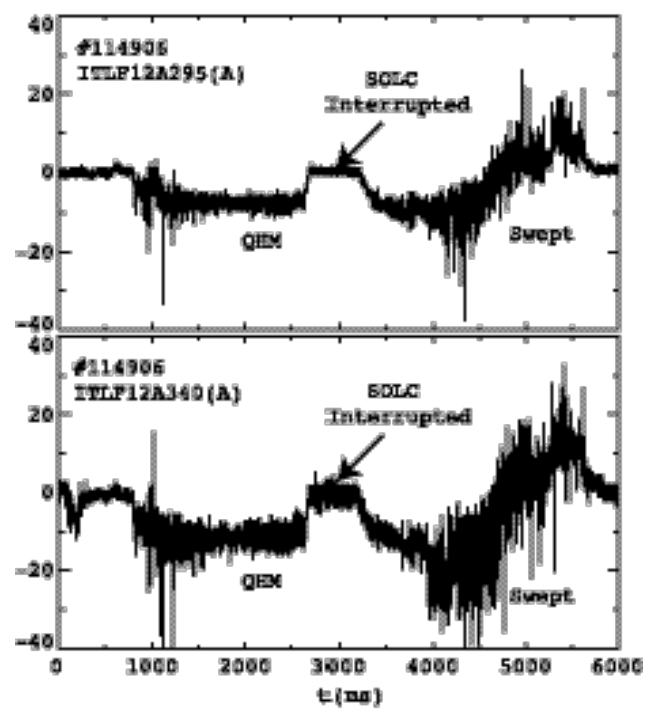

Fig. 4 Signals from tile current sensors in the tile ring \#12A in the discharge shown in the previous figure. It has a period of QHM over 1550-3800 ms. The upper outboard strike point is "swept" over 3200-4900 ms. During the period 2700-3200 ms the signals are essentially zero because the gap leading to the divertor plates is closed off by the neighboring baffle plate \#13A.

The SOLC is small in this discharge, especially during the QHM period, in comparison with that in discharges with MHD activity (see below). The "DC component" (time average over 2200-2400 $\mathrm{ms}$ ) is $-7.6 \mathrm{~A}$ and $-10.8 \mathrm{~A}$ for ITLB295B and ITLB340B signals, respectively. The measured currents vary by $\pm 17 \%$ of average value of $-9.2 \mathrm{~A}$ of these signals. But the variation is within the estimated uncertainty in the sensor sensitivity. The SOLC is toroidally symmetric, within the uncertainty of the measurement, and the total current involved is small, on the order of $0.5 \mathrm{kA}$ (48 times $9.2 \mathrm{~A}$ ), in this quiescent discharge period.

The SOLC observed in the quiescent discharge shown in Fig. 4 appears to be consistent with a thermoelectric potential origin. The current during this discharge period (2200-2400 ms) flows into 
only a $2 \mathrm{~cm}$ wide (radially) part of the tile. (This is not because the radial profile of the current density is inherently narrow, but because the gap leading to the divertor region is only slightly open - see Fig. 23 in Sec. 6.3.) Taking into consideration the toroidal tile width of about $18 \mathrm{~cm}$ and a calculated angle of $3.3^{\circ}$, with which field-lines intersect the divertor plates ("grazing field-line angle"), the measured 9.2 A current corresponds to the density of current flowing parallel to field lines ("parallel current density") of $4.4 \mathrm{~A} / \mathrm{cm}^{2}$. Measurement by the TS diagnostic in a region of the outboard SOL above the mid-plane $(\mathrm{R}=194 \mathrm{~cm}$ and $\mathrm{Z}=75 \mathrm{~cm})$ showed that $\mathrm{T}_{\mathrm{e}} \sim 90 \mathrm{eV}$ and $\mathrm{n}_{\mathrm{e}} \sim 4 \times 10^{12} / \mathrm{cm}^{3}$ at the separatrix at $2300 \mathrm{~ms}$. The electron temperature at the divertor plates was not measured in this discharge. However, measurement in a USN discharge from the same series of experiments, but at slightly lower $\mathrm{I}_{\mathrm{p}}\left(\right.$ by $\sim 8 \%$ ), showed $\mathrm{T}_{\mathrm{e}}$ and $\mathrm{n}_{\mathrm{e}}$ of $\sim 78 \mathrm{eV}$ and $\sim 1.8 \times 10^{13} / \mathrm{cm}^{3}$ at the outboard divertor plates and $\sim 27 \mathrm{eV}$ and $\sim 2.4 \times 10^{13} / \mathrm{cm}^{3}$ at the inboard divertor plates, with a $\mathrm{T}_{\mathrm{e}}$ difference of $\sim 51 \mathrm{eV}$. The parallel current density estimated from theory [16] for these parameters was $\sim 5.5$ $\mathrm{A} / \mathrm{cm}^{2}$, in approximate agreement with the measured value. A current density profile under conditions analogous to this measuring time point (2300 ms) appears in Fig. 24 (green curve).

The parallel current density $\left(4.4 \mathrm{~A} / \mathrm{cm}^{2}\right)$, measured in this H-mode USN discharge with the ion $\square \mathrm{B}$ drift direction away from the X-point, was comparable to, or somewhat smaller than, past measurements in large tokamaks. The current density was $5 \mathrm{~A} / \mathrm{cm}^{2}$ in an L-mode LSN discharge in DIII-D in which the measured outboard (hotter side) divertor $T_{e}$ was $30-40 \mathrm{eV}$ and an estimated $T_{e}$ difference was 20-30 eV [5]. A peak (spatial) parallel current density of $\sim 9 \mathrm{~A} / \mathrm{cm}^{2}$ was reported for L-mode attached divertor discharges in JT60-U in which $\mathrm{T}_{\mathrm{e}} \sim 55 \mathrm{eV}$ in the outboard divertor and $\sim$ $25 \mathrm{eV}$ in the inboard divertor [11]. The ion $\square \mathrm{B}$ drift direction was toward the $\mathrm{X}$-point in both cases.

Coherent and persistent oscillations with small amplitude are observed also in the SOLC signals during the QHM period in this discharge. These oscillating SOLCs are of some interest because of the potential significance attached to the EHO in investigation of the origin of the QHM regime. The spectral characteristics of these SOLC oscillations are similar to those of EHOs detected by Mirnov coils. Spectral peaks, up to the fourth harmonic, are recognizable in the plot of the modulus of fast Fourier transform (FFT) vs. frequency of both the SOLC and Mirnov coil signals. The second harmonic peak at $16.15 \mathrm{kHz}$ is the largest, followed by the fundamental at $8.05 \mathrm{kHz}$, and is toroidally non-axisymmetric by a factor of two or more in both the Mirnov and SOLC signals. The observed non-axisymmetry of the oscillation amplitude, which is well outside the estimated uncertainty in the sensor sensitivity for either diagnostic, is in contrast to expectations from a simple model of a rotating global MHD mode. The observed SOLC oscillation amplitude was small (a few A per tile), possibly because only part of the current was intercepted by the divertor tile (more on this in Sec. 6.3).

The discharge described in Figs. 3 and 4 serves a second purpose in providing a measurement of the radial profile of a SOLC in a quiescent discharge period. To this end, the upper outboard separatrix strike point, which falls on the tile ring \#12A, is moved radially ('swept') over the time range, 3200-4900 ms. Both signals in Fig. 4 are negative over most of the discharge duration (except for the period, 2700-3200ms, over which they are nearly zero). But they reverse their signs near the end of the discharge. This can be interpreted as the result of the swept strike point, coupled with a radial profile of the SOLC with a polarity reversal as a function of the radial distance. This will be discussed further in Sec. 6.3.

\subsection{SOLC in discharges with ELMs}

Large SOLCs are commonly observed in discharges with ELMs ('ELMing' discharges) in DIII-D and in many other devices. Figure 5 shows tile current signals in a discharge that was specifically designed to generate a long series of ELMs, and had $\mathrm{B}_{\mathrm{t}}=-1.75 \mathrm{~T}, \mathrm{I}_{\mathrm{p}}=+1.39 \mathrm{MA}, \mathrm{P}_{\mathrm{b}}=$ $8 \mathrm{MW}$, and $\square_{\mathrm{N}} \sim 2$. The ion $\square \mathrm{B}$ drift was downward, toward the X-point, in this LSN discharge with $B_{t}<0$. The field lines have lefthanded twist in this standard DIII-D magnetic configuration. One signal (top panel) is from a tile 
in the slanted ring \#10B in the inboard divertor and the other (bottom panel) is from a tile in the ring \#13B in the outboard divertor. The inboard and outboard separatrix strike points fall on these two rings, and the two divertors are connected to each other through common flux over a significant radial width. The tiles in the two rings have a different toroidal angular width $\left(7.5^{\circ}\right.$ for the inboard tiles and $5^{\circ}$ for the outboard tiles).

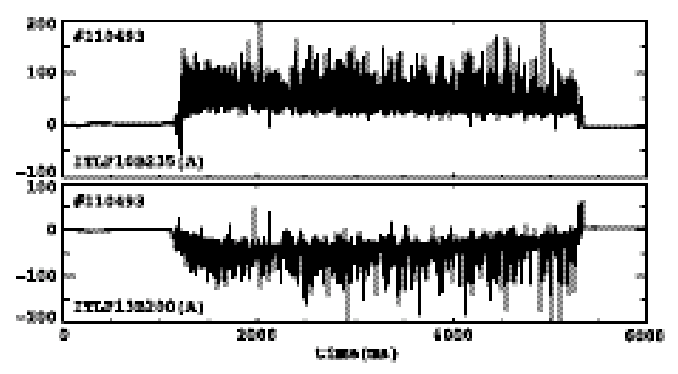

Fig. 5 Tile current sensor signals from the bottom divertor in an LSN H-mode discharge with ELMs, (top panel) one from the inboard slanted tile ring \#10B and (bottom panel) the other from the outboard ring \#13B. A positive signal means a current flowing into the tile from the SOL, and vise versa. The current flows from the outboard to inboard divertor in this discharge.

The SOLC shown in Fig. 5 flows along open field lines from the outboard divertor to the inboard divertor. The signal is mostly in the negative domain for the outboard sensor and in the positive domain for the inboard sensor. The current is measured directly only as it flows between the tiles and the vessel, and nowhere else along the field lines. But opposite signs of the signals from the tile rings connected through field lines support the notion that the current indeed leaves the outboard ring, flows along open field lines, and then enters the inboard ring. There are many other pieces of circumstantial evidence observed in a number of tokamaks to buttress this notion. "Shadowing" of the SOLC by a protruding neighboring tile ring is an example (see Sec. 6.3). Nevertheless, a "total quantitative accounting" of the SOLC, i.e., total current measured on the outboard rings equaling the negative of total current measured on the inboard rings, is found difficult to accomplish in this and other [5] discharges in DIII-D as well as discharges in other devices [11]. Insufficient spatial resolution of the measurement is one reason for the difficulty. Complex current path patterns, such as those with polarity reversal (see Sec. 6.3), may be another. In experiments with actively driven SOLCs, current paths other than fieldaligned ones were postulated to explain the experimental observations [32].

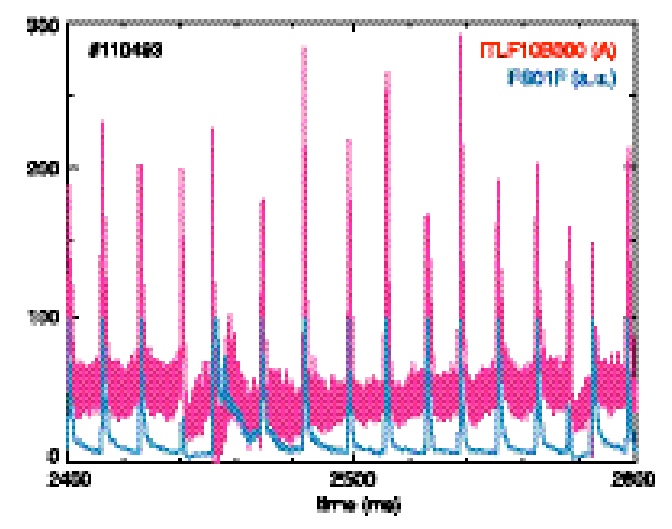

Fig. 6 An expanded view of the signal from a tile current sensor (magenta) in the inboard slanted ring $\# 10 B$, together with a $D_{\square}$ light signal (cyan), obtained in the ELMing discharge shown in the previous figure. Spikes in the tile current signal are as good an indicator of ELMs as those of a $D_{\square}$ light signal. SOLC during some ELMs reach nearly $300 \mathrm{~A}$ in a single tile in this discharge.

With field lines of left-handed twist the signs of the signals in Fig. 5 imply that the SOLC flows toroidally in the same direction as the plasma current in this discharge. Assuming that $\mathrm{T}_{\mathrm{e}}$ was higher in the outboard divertor than in the inboard divertor in this discharge, as is often the case in this magnetic configuration (ion $\square \mathrm{B}$ drift toward the X-point) in DIII-D, the current flowed in the same direction as the thermoelectric potential gradient, and is in agreement with earlier findings [4ฤ6].

Figure 6 shows spiky features in a tile current signal (magneta) from the inboard ring \#10B in the same discharge shown in the previous figure (but a different tile). $\mathrm{A}_{\square}$ light signal (cyan) measured near the inboard strike point is also shown. Spiky features in both traces are coincident with each other. SOLC spikes can thus be as good an indicator of the occurrence of ELMs as $\mathrm{D}_{\square}$ light spikes in this discharge. This has been found to be the case in many other ELMing discharges examined in DIII-D. The "fuzzy" nature of the SOLC trace between ELM spikes is from 
persistent moderate-amplitude oscillations at a few tens of $\mathrm{kHz}$ that cannot be resolved on this time scale.

The SOLC reaches nearly $300 \mathrm{~A}$ in a single tile during some ELMs as shown in Fig. 6, and current of up to $400 \mathrm{~A}$ in a single tile has been observed in other discharges [9]. The SOLC tends to be more or less axisymmetric at the peak of an ELM [7-9]. The total current involved may reach $20 \mathrm{kA}$ at the peak. But its magnetic consequences may not be as significant as this number may suggest because of its axisymmetry. The SOLC has been observed to be more strongly non-axisymmetric [6-9] in the early phase of the ELM in DIII-D. The current has in fact been found to begin to rise already during the precursor period of ELMs in TCV [13] and DIII-D [9]. Assessing the magnetic consequences of this smaller but non-axisymmetric SOLC is a goal of ongoing and future research. A body of information has been compiled on the SOLC associated with the ELM in DIII-D. But it would be too lengthy to be included in its entirety in this article, which covers a range of MHD activity. It will be discussed in a separate article for the ELM.

\subsection{Large amplitude oscillating SOLC}

The observation of coherently oscillating SOLC may be a surprising discovery. Small and moderate amplitude oscillations have already been referred to in connection with the EHO and the ELM, respectively. Oscillating SOLCs are fairly ubiquitous in discharges in DIII-D, and possibly also in other devices, but have not been previously reported, perhaps because the SOLC has not been routinely observed at a high enough time resolution. The data here is sampled at $200 \mathrm{kHz}$.

Large amplitude oscillating SOLCs are sometimes found in discharges with $\square_{\mathrm{N}}$ comparable to, or higher than the nominal no-wall limit for the kink mode. Figure 7 is a pictorial summary of the conditions of a discharge in which $\mathrm{I}_{\mathrm{p}}$ is ramped up (here, $\sim 0.25 \mathrm{MA} / \mathrm{sec}$ ) to generate edge current and lower the $\square$ limit for kink modes. Operating regimes above the no-wall limit can be accessed readily in this type of discharge for investigations of the MHD stability. This discharge undergoes a few cycles of thermal collapses followed by recoveries. It is interesting to note in this and other similar cases that the discharge is often able to recover from a thermal collapse. The SOLC is also quenched at a thermal collapse (see next section). Large amplitude oscillating SOLCs are seen during a period leading to each thermal collapse.

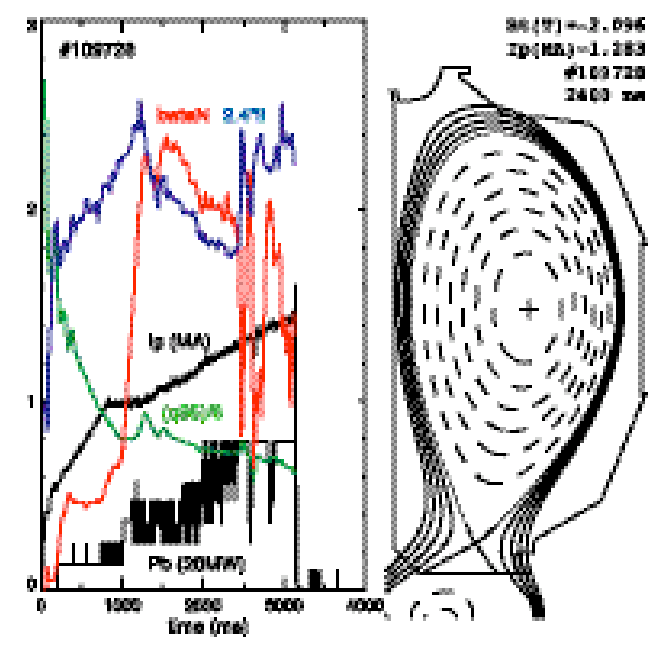

Fig. 7 A Pictorial discharge summary for examination of large amplitude oscillating SOLC (see caption to Fig. 3 for explanation). Edge current generated by $I_{p}$ ramp $(\sim 0.25 \mathrm{MA} / \mathrm{sec})$ lowers the $\square$ limit, and makes operating regimes above no-wall limit readily accessible for study.

Large oscillations occur in the SOLC, together with those in magnetic field and $\mathrm{T}_{\mathrm{e}}$ that are comparable in amplitude as global MHD modes. Figure 8 shows oscillations in tile current measured on the inboard slanted ring \#10B (top panel), $\mathrm{T}_{\mathrm{e}}$ at a mid-radius $(\square=0.44$ where $\square$ is normalized minor radius based on toroidal flux) measured by ECE (middle panel), and poloidal magnetic field measured by a Mirnov coil in the outboard mid-plane (bottom panel). The period covered in the figure is between the first and second thermal collapses shown in Fig. 7 (red curve). Regular oscillations in the SOLC signal are interrupted over two brief intervals, 2576.3-2577 $\mathrm{ms}$ and 2582.4-2583.3 ms, during the period shown in the figure. The nature of these "disturbances," which punctuate the persistent saturated oscillations at irregular intervals, is not understood, but believed to be a phenomenon separate from the regular oscillations. (Several similar events shown on a longer time scale are recognizable in Fig. 13, panels (a) and (b)). The regular oscillations in all three signals in the figure are at about $5.5 \mathrm{kHz}$ and 
phase-locked with each other. (The regular oscillations of up to $12 \mathrm{kHz}$ are observed earlier in the discharge.) The peak-to-peak amplitude of the oscillating SOLC reaches $80 \mathrm{~A}$ in a single tile. The total current involved is estimated to be about 1.3 $\mathrm{kA}$. The peak-to-peak $\mathrm{T}_{\mathrm{e}}$ oscillation amplitude reaches $0.5 \mathrm{keV}$. Magnetic oscillations are nearly $250 \mathrm{~T} / \mathrm{sec}(7.2 \mathrm{mT})$ peak-to-peak, comparable to the amplitude of large global MHD modes observed on DIII-D.

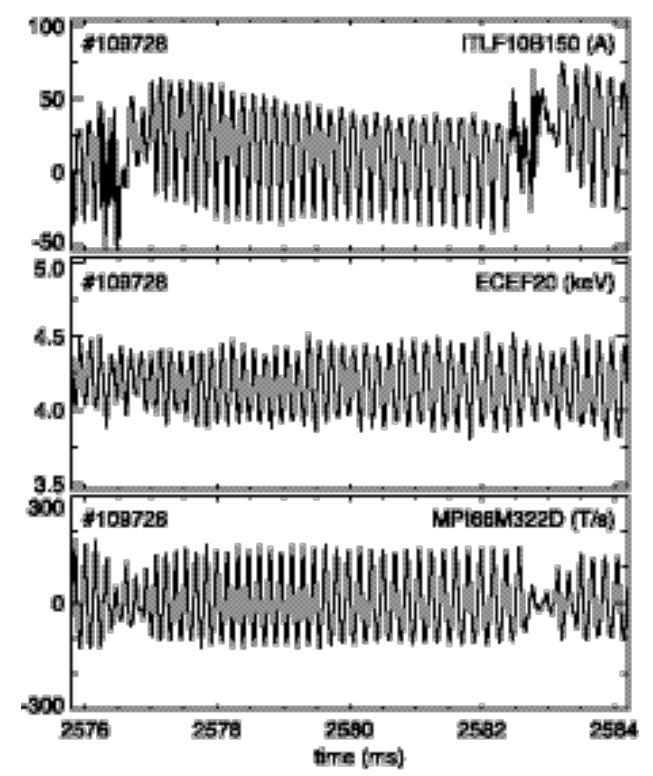

Fig. 8 Oscillating SOLC in the discharge shown in the previous figure: (top) a tile current signal from the inboard slanted tile ring \#10B in the lower divertor, (middle) $T_{e}$ at a mid-radius $(\square=0.44)$ measured by the ECE diagnostic, and (bottom) $B_{\square}$ dot signal from a Mirnov coil in the outboard mid-plane. Magnetic, $T_{e}$, and SOLC perturbations are at $\sim 5.5 \mathrm{kHz}$ and phaselocked. The spatial structure of both SOLC and Mirnov oscillations is $n=1$ (see Figs. 13 and 14).

The observed SOLC is toroidally bi-polar, and yields a clue as to its potentail origin. It will be shown in the next section that the oscillations in the SOLC signal in Fig. 8 (top panel) represent toroidally traveling perturbations. Its temporal variation, ranging in both positive and negative domains, then implies that the current was of one sign at some points in the torus while it was of an opposite sign at other places at the same instant in time. This feature makes the EMF of MHD activity a candidate mechanism for driving the observed SOLC. However, it does not necessarily rule out a thermoelectric origin: MHD activity, or some other causes, may have somehow modulated the toroidal $\mathrm{T}_{\mathrm{e}}$ variation in such a way as to drive a bi-polar SOLC, and the resultant non-axisymmetric SOLC pattern may have rotated to produce the observed oscillations.

\subsection{Large SOLC Driven by Flux Swing}

Very large spiky SOLCs are often observed during a thermal collapse and a partial (or complete) $I_{p}$ quench that frequently follows the collapse. The discharge shown in Fig. 9 is, like the one in Fig. 7, also intended for MHD stability studies, but $\mathrm{I}_{\mathrm{p}}$ is ramped up faster here $(\sim 1 \mathrm{MA} / \mathrm{sec})$. A significant "dip" is recognizable in the $\mathrm{I}_{\mathrm{p}}$ trace around 1800 ms as a result of a current quench.

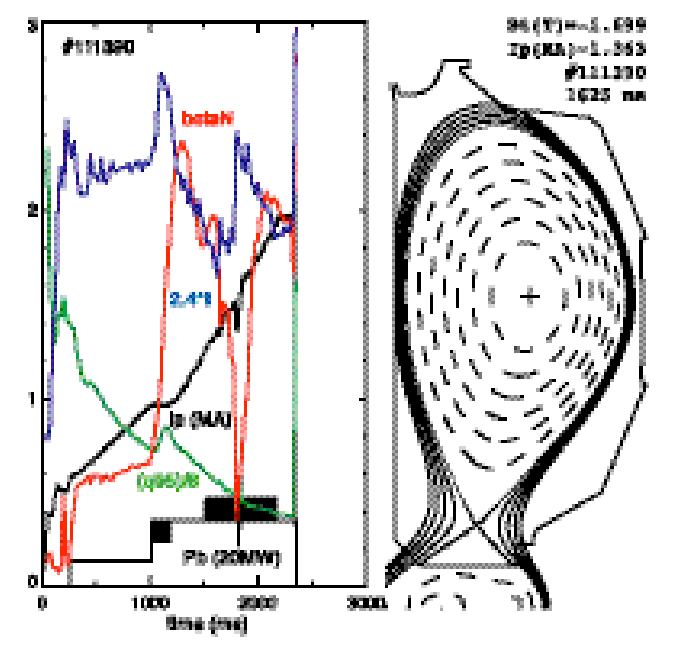

Fig. 9 A pictorial discharge summary for examination of large spiky SOLC (see caption to Fig. 3 for a figure format explanation). Discharge similar to the one in Fig. 7, but at lower $q$-values, with lower $B_{t}$ and faster $I_{p}$ ramp up $(\sim 1 \mathrm{MA} / \mathrm{sec})$.

This discharge demonstrates that the SOLC can be a dynamic source of error field. SOLC signals are shown in Fig. 10 for a period containing thermal collapses and current quenches. Both signals are from tiles on the slanted ring $\# 10 \mathrm{~B}$, one at $0^{\circ}$ toroidal angle and the other at $150^{\circ}$. A large spike in excess of $600 \mathrm{~A}$ in the $0^{\circ}$ signal (top panel) occurs at about $1640 \mathrm{~ms}$, but no comparable size spike occurs in the $150^{\circ}$ signal (bottom panel). The several-fold difference between the SOLC amplitudes at these two toroidal locations during 
this spike is well outside the uncertainty in the sensor sensitivity, and the observed SOLC is thus strongly non-axisymmetric. The roles of these signals are reversed later in the discharge: a spike in excess of $800 \mathrm{~A}$ around $1790 \mathrm{~ms}$ in the $150^{\circ}$ signal has no counter-part in the $0^{\circ}$ signal. The peak SOLC thus does not always occur at the same toroidal location, even within the same discharge an observation that is independent of the sensor sensitivity. This demonstrates that the observed non-axisymmetry is not a result of permanent structural features of the device, such as unevenly laid divertor tiles or error field produced by imperfect coils. The SOLC can thus be a source of error field that varies dynamically, within the discharge as well as from one discharge to another.

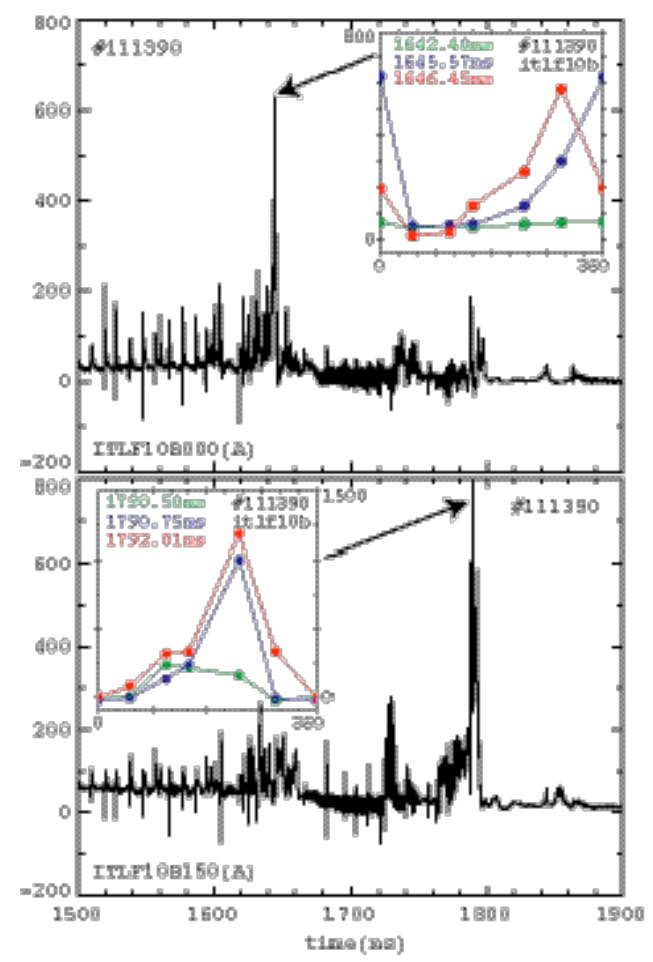

Fig. 10 SOLC signals from two tiles at $0^{\circ}$ and $150^{\circ}$ toroidal angles on the slanted ring \#10B in the discharge described in the previous figure. The SOLC is toroidally non-axisymmetric at many temporal spikes. The peak value also does not always occur at the same toroidal location, even in the same discharge. Inset in each panel shows toroidal variation of the SOLC at 3 time points around the peak indicated by an arrow.

The observed SOLC is toroidally uni-polar, and suggests its potential origin. The inset in each panel of Fig. 10 shows the toroidal variation of the SOLC, constructed from all six available sensor signals (two of which are presented in the main plots) at three time points around the peak shown in each panel. The SOLC signals are largely positive everywhere around the torus at these time points. This may be an indication that the observed SOLC was driven mainly by the flux swing of the plasma column, rather than directly by the EMF of the MHD activity. But the thermoelectric potential generated by a toroidally non-axisymmetric $T_{e}$ distribution cannot be ruled out.

The SOLC can vary in magnitude and distribution very quickly. The toroidal profile of the observed SOLC rotated quickly by about $75^{\circ}$ within a sub$\mathrm{ms}(0.88 \mathrm{~ms})$ interval (from blue to red curve in top inset of Fig. 10). The peak (spatial) current rose from $0.5 \mathrm{kA}$ to $1 \mathrm{kA}$ in a sub-ms $(0.25 \mathrm{~ms})$ interval (from green to blue curve in bottom inset). The SOLC reached $1.2 \mathrm{kA}$ (red curve in bottom inset), with an estimated total current involved of $20 \mathrm{kA}$ (integration of the "area under the curve" yields a similar magnitude). Even larger SOCs were observed during a thermal collapse, followed by a current quench, in other discharges that exceeded the limits (1.5-2 kA for most channels) of measurement channels.

Other magnetic consequences may arise from toroidally non-axisymmetric field generated by the SOLC when detected by toroidally discrete magnetic sensors, such as Mirnov coils and saddle loops. It may be falsely interpreted as axisymmetric field by the tokamak control logic and in equilibrium reconstruction routines.

\section{Estimating Magnetic Consequences of SOLC}

\subsection{Need for knowledge of SOLC structure}

The SOLC may serve as a flux converter, generating helical flux at the expense of axisymmetric one. On the one hand, the potential for generating a helical error field rests in the nonaxisymmetry in the toroidal distribution of the SOLC. This potential may be enhanced when the SOLC circuit topology has significant inductive 
coupling to the plasma column. While magnetic flux, both toroidal and poloidal, of the plasma column is axisymmetric. it can generate a helical error field when combined with the SOLC's demonstrated propensity to flow in a nonaxisymmetric pattern. On the other hand, a number of factors may mitigate this potential. A radially distributed current experiences "phase mixing," as explained in more detail below. A return current path running closely parallel to the forward path makes a current circuit a dipole-like source of magnetic field, which decays rapidly with distance. More complex circumstances may exist, involving multiple current paths inside the SOL. Both enhancing and mitigating potentials are strongly influenced by the SOLC structure. Important SOLC characteristics are tabulated here:

(a) Current intensity

(b) Current density distribution

i. Toroidal (non-axisymmetric field)

ii. Radial (phase mixing)

(c) Current path topology

i. Return path (field cancellation)

ii. Circuit geometry (inductive coupling).

\subsection{Models for SOLC}

A line current model forms an elementary basis for examining the SOLC in a complex situation. The model is based on the assumption that the SOLC flows along field lines (force-free current). A distributed current may be represented in terms of summation over line currents with different intensities and topological characteristics. But studying a single line current in itself provides an insight into the importance of the circuit topology.

Figure 11 (left panel) shows a 3D rendering of an open field line (red helix) traced in the SOL of an equilibrium computed for a USN configuration in DIII-D. The field line strikes tile surface at some points (blue dots). The current is assumed to flow along the field line, enter the tile at one of the strike points, flow through the tokamak structure, emerge at the other strike point to close its circuit. The current path inside the structure (green curve) is chosen arbitrarily. The figure also shows the plasma boundary and flux surfaces, both in a 2D cross-sectional rendering, the magnetic axis (gray circle), and the directions (arrows) of $\mathrm{B}_{\mathrm{t}}$ and $\mathrm{I}_{\mathrm{p}}$. The SOL field line shown here passes through a point that is $1 \mathrm{~cm}$ outside the plasma boundary in the outboard mid-plane. It executes 6.75 toroidal revolutions between the two strike points, indicating that the "effective average q-value" is nearly 7. Note that an open field line does not need a rational q value for its circuit to close on itself.

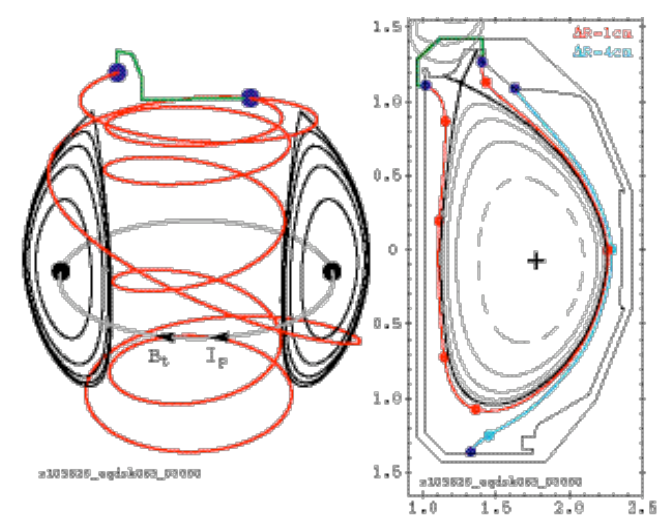

Fig. 11 A line-current model of the SOLC: (left) $3 D$ schematic rendering of a SOLC circuit in a USN discharge in DIII-D; the red helical curve represents the path along an open field line; the green curve is the path within the vacuum vessel walls; the two join each other at divertor tiles represented by the blue dots. Shown schematically in $2 D$ rendering are the plasma boundary, flux surfaces, and the magnetic axis (in gray): (right) a flux plot of the equilibrium used to construct the configuration in the left panel. $2 D$ poloidal projection (red and green curves), together with puncture points (red dots) is shown for the $3 D$ circuit in the left panel. Another SOL field line (cyan) is also shown that passes a point in the outboard midplane $3 \mathrm{~cm}$ father out than the field line in red.

An open field line in the SOL contains a wide spectrum of q-values. Figure 11 (right panel) is a poloidal flux plot of the equilibrium used to construct the 3D configuration in the left panel. A 2D poloidal projection (red curve), together with puncture points (red dots), is also shown for the SOL field line given in the left panel. Inspection of the puncture point spacing, or more directly from a plot (not shown) of the field line in a $\mathrm{CD}$ plane ( $\square$ and $\square$ are poloidal and toroidal angles, respectively) indicates that the field line pitch angle is close to unity (local $\mathrm{q} \equiv d \square \square d \square \sim 1$ ) when averaged over $\square= \pm 60^{\circ}$ about the outboard mid- 
plane, and about four (local q 4) over a wider $\square=$ $\pm 90^{\circ}$ range. Nearly four toroidal revolutions occur on the inboard side with a local $\mathrm{q} \sim 8$.

A small change in the field line path can result in drastic changes in the SOLC circuit topology and presumably its magnetic consequences. A poloidal projection (cyan curve) of an SOL field line, located $3 \mathrm{~cm}$ farther out in the SOL in the outboard mid-plane than the first field line (red curve), is also shown in Fig. 11 (right panel), together with puncture points (cyan dots). With its separatrix strike points (blue dots) at the top and bottom of the chamber, this field line (cyan) traces a trajectory very different from the first field line (red). Return current path through the structure is also likely to differ significantly.

The non-axisymmetry of SOLC at the divertor plates assures generation of non-axisymmetric error field, as long as the current thickness is infinitesimal in the radial direction. As a next higher level of approximation of the SOLC structure, a sheet current model may be constructed from multiple line currents. A sheet current with a non-axisymmetric toroidal density variation is a basis for generating non-axisymmetric error field. In this approximation, the toroidal "phase" of the variation, measured in the divertor on a horizontal circle drawn on a tile ring, is mapped one-to-one to a horizontal circle at any other point on the same flux surface, including, for example, the outboard mid-plane, with only a rigid-body rotation of the toroidal pattern as a whole.

The efficacy of the SOLC in generating error field may be mitigated in a more realistic raidally distributed current. A model of the SOLC may be constructed from multiple sheet currents distributed over a radial distance. These sheet currents undergo in general a different degree of rotation at different radial positions, with consequent mixing of the phase of the toroidal patterns - effect studied qualitatively using the line current model in the next sub-section.

\subsection{Phase mixing}

Because of the shear in the field line pitch angle in the SOL region, field lines originating at different radial locations on a divertor tile go through a different number of toroidal revolutions before reaching another poloidal location. The SOLC, distributed non-axisymmetrically at each radial location on a divertor tile, could thus appear toroidally axisymmetric at another poloidal location because phases of many toroidal variations are mixed together upon integration over a finite radial width. In order to calculate the nonaxisymmetric field from a measured SOLC, it is necessary to include the potentially important effect of "phase mixing."

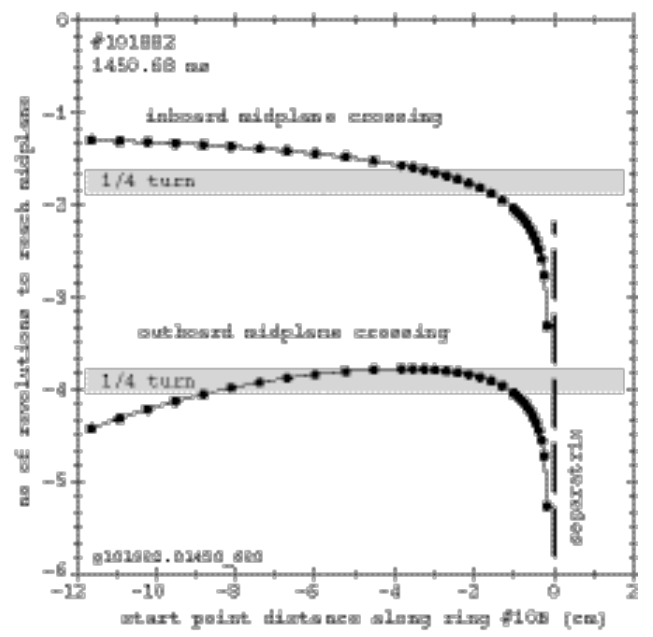

Fig. 12 A measure of the phase mixing effect is shown: the number of toroidal revolutions that an open field line executes before reaching either the inboard or outboard mid-plane, starting from a point on the slanted $\# 10 B$ tile ring, is plotted against the distance to the starting point from the separatrix strike point. A region of rapid variation next to the strike point and a broad plateau are evident. Two gray bands indicate a 1/4 revolution.

A measure of the phase mixing effect may be constructed in terms of the variation of the number of toroidal revolutions to reach a given poloidal angle, as a function of the starting point radial location. Shown in Fig. 12 is a "number of toroidal revolutions" plot for an LSN discharge. The field line starts from a point on the slanted \#10B tile ring, and the number of toroidal transits is calculated as the field line travels poloidally in the SOL. The plot shows the number of revolutions recorded when the field line reached either the inboard or outboard mid-plane as a function of the starting point location. The number of revolutions 
is shown as a negative number because the field line runs in a clock-wise direction (negative toroidal direction). The starting point location is its distance from the inboard separatrix strike point measured along the tile ring surface, and is also shown as a negative number as the starting point lies on the smaller major radius side of the strike point.

The SOLC flowing close to the separatrix is ineffective in generating a toroidally nonaxisymmetric error field. As seen in Fig. 12 the number of toroidal revolutions varies strongly when the starting point is within about $1 \mathrm{~cm}$ of the separatrix strike point as measured on the tile surface. This distance corresponds to about $0.4 \mathrm{~cm}$ in the outboard mid-plane, making the "flux expansion ratio" from the outboard mid-plane to the inboard divertor about 2.5 in this equilibrium. Field lines originating from this high-shear radial zone have a strongly varying toroidal phase when they reach the outboard mid-plane. Field produced by the SOLC flowing along these field lines in the strong phase-mixing zone tends to be toroidally axisymmetric.

But the SOLC can also generate toroidally nonaxisymmetric field. Perhaps surprising is the presence in Fig. 12 of a broad plateau in the curves outside the strong phase mixing zone, especially in the "outboard mid-plane curve." Each of two gray horizontal bands in the figure represents a region where the variation is less than $1 / 4$ revolution. Field lines originating from points at identical toroidal angles, but different radial locations between about $1 \mathrm{~cm}$ and $9 \mathrm{~cm}$ from the separatrix strike point, retain their toroidal phase within about $1 / 4$ turn when they reach the outboard mid-plane. This low-shear, weak phase-mixing radial zone is between about $0.4 \mathrm{~cm}$ and $2.2 \mathrm{~cm}$ when maped to the outboard mid-plane. Current flowing along these field lines is likely to be the most effective in generating an error field on the outboard midplane, where it is also expected to be most effective in interacting with MHD modes.

\section{SOLC and MHD Activity}

The SOLC is an integral part of MHD activity, not an isolated phenomenon. This can be demonstrated by examining the SOLC in the context of other common signatures of MHD activity.

The toroidal non-axisymmetry and other characteristics are examined for SOLCs correlated with three types of MHD activity: (a) slowly growing magnetic perturbations in a LM-like process, (b) large amplitude traveling kink-like oscillations, and (c) a fast thermal collapse followed by a current quench. The discharge shown in Fig. 7 is used to study both the LM-like process and kink-like oscillations. A second discharge is analyzed for a fast thermal collapse and current quench. Both are discharges with $\square_{\mathrm{N}}$ above the nominal no-wall limit, a regime in which various forms of MHD instability, including the RWM, NTM and LM, are presently thought to limit the plasma performance.

\subsection{SOLC in slow MHD activity}

Examinations of slow MHD activity in this subsection serve to demonstrate the existence of non-axisymmetric SOLCs that are demonstrably not driven by the EMF of the MHD activity.

A non-axisymmetric SOLC can appear in close correlation with a LM-like sequence of events in other discharge parameters. Shown in Fig. 13 are the time variations of some parameters of interest over parts of the discharge shown in Fig. 7. The panels, (a) and (b), show SOLCs in the slanted ring $\# 10 \mathrm{~B}$ at toroidal angles of $0^{\circ}$ and $150^{\circ}$, respectively, in units of Amperes. The panel (c) describes the signal (red) from a "locked-mode detector" (saddle loop that detects radial magnetic field, $B_{r}$ ) in units of Gauss and the signal (blue) from the CER diagnostic showing the toroidal rotation speed of a carbon impurity species $\left(\mathrm{C}^{+6}\right)$ at a mid-radius location in units of $10 \mathrm{~km} / \mathrm{s}$. The panel (d) is $T_{e}$ at a mid-radius location $(\square=0.44)$ measured by the ECE diagnostic in units of $\mathrm{keV}$. The panel (e) shows the signal from a Mirnov coil in the outboard mid-plane, measuring the timevariation of the poloidal magnetic field, $\mathrm{B}_{\square}$ dot, in units of $\mathrm{T} / \mathrm{sec}$.

Page 15 of 29 
These signals in Fig. 13 together exhibit the hallmarks of a LM sequence of events. Dark areas in the SOLC, ECE, and Mirnov traces are oscillations in the range of several $\mathrm{kHz}$ that cannot be resolved on the time scale of the plots. Parts of these signals were presented earlier in Fig. 8 at a higher time resolution (see also next subsection). The Mirnov signal exhibits the temporal behavior of a mode-locking process, with its amplitude decreasing from a broad maximum of about 300 T/sec peak-to-peak around $2570 \mathrm{~ms}$ toward essentially zero (mode locking) at $2595 \mathrm{~ms}$. The toroidal rotation speed also slows down, from about $100 \mathrm{~km} / \mathrm{sec}$ to essentially zero over the same period. The locked-mode signal begins to rise at the time of the mode locking, and grows slowly to a peak value of about $13 \mathrm{G}$ at $2607 \mathrm{~ms}$. $\mathrm{T}_{\mathrm{e}}$ collapses at this time point.

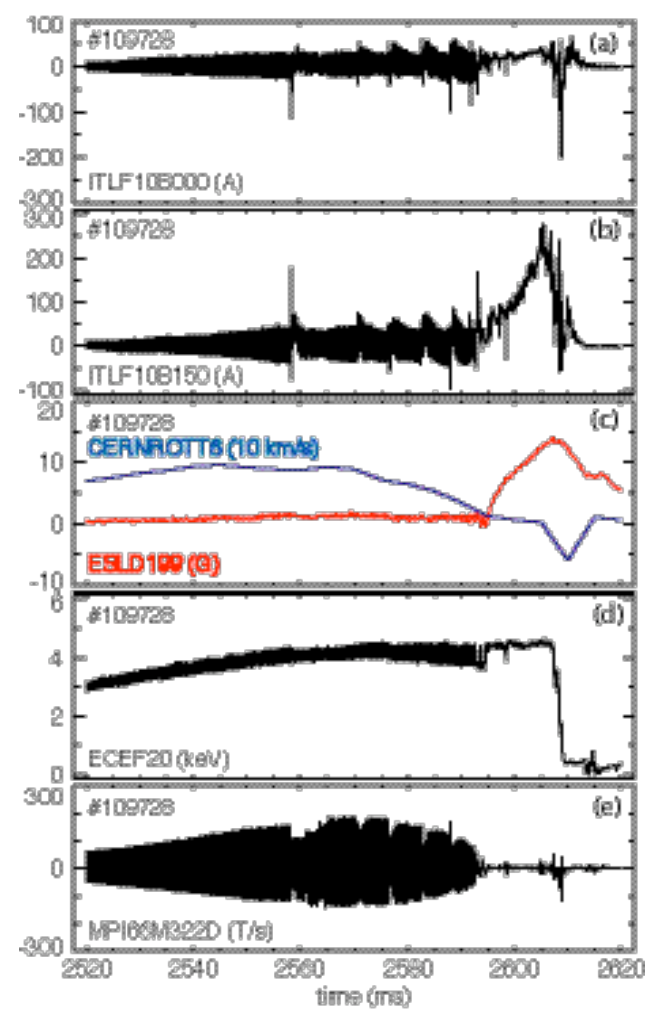

Fig. 13 Signals of interest from a discharge with a LM sequence: (a)-(b) tile current sensors, (c) $B_{r}$ from a locked-mode detector (red) and carbon rotation speed (blue), (d) $T_{e}$ at $\square=0.44$, and (e) Mirnov coil on outboard mid-plane. Growing non-axisymmetric SOLC appears, beginning at about 2595 ms. Oscillations (dark areas) from this discharge were shown in Fig. 8 on expanded time scale.
The focus of this subsection is the period after the mode locking. The SOLC at both locations ceased to oscillate at the time $(\sim 2595 \mathrm{~ms})$ of the mode locking. The SOLC at $150^{\circ}$ increases strongly and secularly thereafter, reaching $270 \mathrm{~A}$ at $2605 \mathrm{~ms}$, and then rapidly diminishes as $\mathrm{T}_{\mathrm{e}}$ collapses. But the SOLC at $0^{\circ}$ barely changed in comparison over the same period. The toroidal non-axisymmetry of the SOLC in this discharge was evident in all six of the available tile current signals in the slanted ring \#10B (two of which are shown in Fig. 13). The toroidal variation was uni-polar, suggesting that the EMF of MHD activity was not among candidate driving mechanisms during this period.

Buttressing this point was the quasi-static nature of the variation of the observed magnetic field (see LM signal (red) in panel (c)) during the period following the mode locking. The peak amplitude of the SOLC during the oscillating period was $\sim 40 \mathrm{~A}$ (Fig. 8, top panel) while the quasi-stationary SOLC after the mode locking peaks at $\sim 270$ A (Fig. 13, panel (b)) - a nearly seven-fold increase. The peak-to-peak amplitude of the Mirnov signal during the oscillating period was $\sim 275 \mathrm{~T} / \mathrm{sec}$ (Fig. 8 , middle panel) while it was $\sim 3 \mathrm{~T} / \mathrm{sec}$ (Fig. 13, panel (e)) after mode locking - a decrease of nearly two orders of magnitude. A semiquantitative analysis based on a coupled circuits model, given in the discussion section below, indicates that the Mirnov signal shown in Fig. 13, panel (e), would have been $\sim 120 \mathrm{~T} / \mathrm{sec}$, corresponding to the SOLC signal of $270 \mathrm{~A}$ at 2605 ms shown in panel (b), if the EMF of the MHD activity were the driving mechanism. The observed Mirnov signal ( $3 \mathrm{~T} / \mathrm{sec})$ was an order of magnitude smaller than the value expected from this analysis. Furthermore, the waveform of the Mirnov signal in panel (e), when examined on expanded scales (not shown), is found not to be proportional to that of the growing SOLC signal in panel (b), as would have been expected if the EMF were the driving mechanism. In this analysis, the amplitude relationship between the Mirnov and SOLC signals during the oscillating period before mode locking was used to estimate the values of unknown circuit parameters.

The SOLC discussed here possesses the potential to be destabilizing influence. The toroidally non- 
axisymmetric quasi-static growth of the SOLC, correlated with a LM-like process, has been observed in other discharges with $\square_{\mathrm{N}}$ comparable to, or higher than, a nominal no-wall limit. A toroidally non-axisymmetric growing SOLC thus appears to be an integral part of the observed MHD process, but is driven by mechanisms other than the EMF of the MHD activity itself. The SOLC is thus not a purely reactive current here, and has the potential for destabilizing MHD modes. The thermoelectric potential is a candidate driving mechanism for this SOLC. The temperature difference at the two ends of SOL field lines, needed to establish the potential, may have come about from transport altered by MHD activity. But $\mathrm{T}_{\mathrm{e}}$ at the divertor target plates was not measured for this discharge or under other similar circumstances.

\subsection{Traveling Oscillating SOLC}

The large amplitude oscillating SOLC and magnetic and $\mathrm{T}_{\mathrm{e}}$ perturbations shown in Fig. 8 are tightly correlated with each other, and thus are likely to be parts of the same MHD activity.

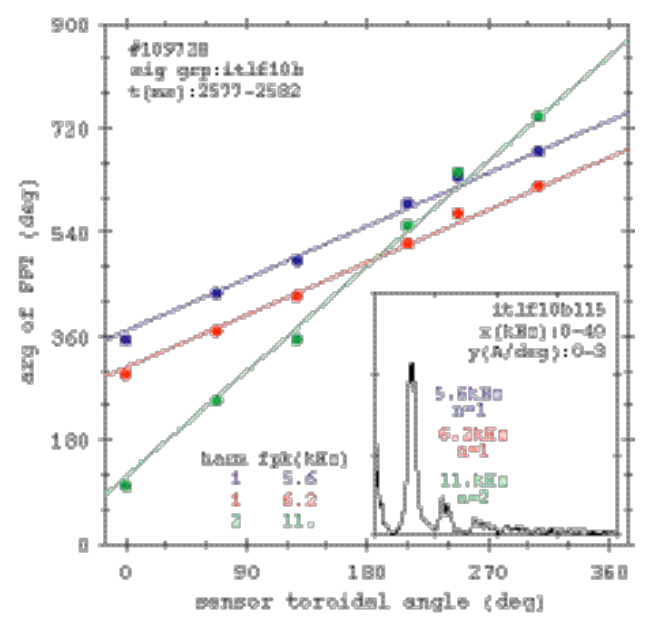

Fig. 14 The toroidal structure of oscillating SOLC is determined from FFT analysis of six sensors in the slanted tile ring \#10B. Inset shows a typical spectrum. Main figure shows the argument of FFT plotted against the sensor toroidal angle to determine a toroidal harmonic number from the slope of a fitted line. Fundamental peaks (blue and red) are an $n=1$, and second harmonic (green) is $n=2$.
Figure 14 summarizes the result of fast Fourier transform (FFT) analysis over the $5 \mathrm{~ms}$ period, 2577-2582 ms, of six oscillating SOLC signals measured by a toroidal array of tile current sensors in the slanted ring \#10B (one of which was shown in Fig. 8). The plot in the inset shows a typical frequency spectrum - the abscissa is frequency, running from 0 to $40 \mathrm{kHz}$, and the ordinate is the current per unit toroidal angle, running from 0 to 3 $\mathrm{A} /{ }^{\circ}$. The fundamental harmonic has two prominent peaks at $5.6 \mathrm{kHz}$ and $6.2 \mathrm{kHz}$. Second harmonic has peaks in the $11.2-12.4 \mathrm{kHz}$ range. The main plot shows the result of a toroidal structure analysis. The abscissa is the location of the sensors, and the ordinate is the argument of FFT of the signals. The slope of a fitted straight line yields a toroidal harmonic number. Both peaks of the fundamental harmonic (blue and red) have an $n=1$ toroidal structure, and one of the peaks examined at the second harmonic (green) has an $\mathrm{n}=2$ structure.

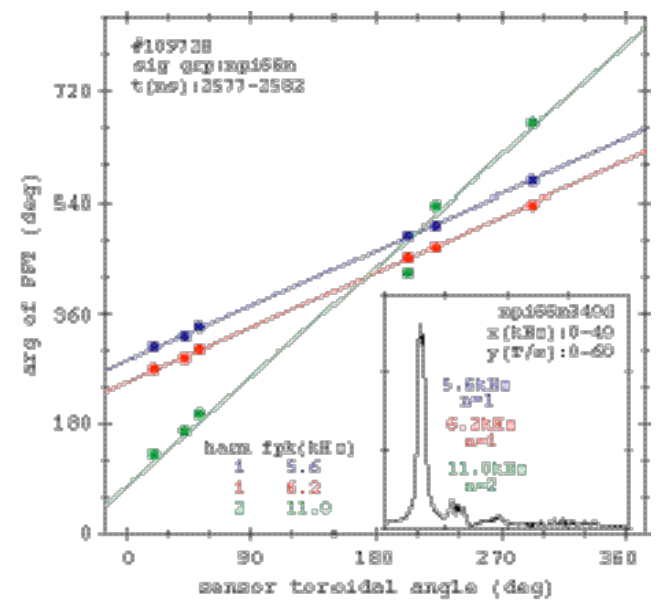

Fig. 15 The toroidal structure of oscillating $B_{\square} d o t$ signals is determined from FFT analysis of six Mirnov coils in the outboard mid-plane. See caption to the previous figure for explanation. Note the similarity of the spectrum and toroidal structure with those of the SOLC in the previous figure.

Figure 15 summarizes the result of an analogous FFT analysis of six oscillating magnetic signals measured by a toroidal array of Mirnov coils in the outboard mid-plane (one of which was shown in Fig. 8). The spectrum typical of the Mirnov signals shown in the inset closely resembles that of the SOLC signals shown in the previous figure, with 
the identical frequencies of the fundamental and second harmonic peaks. Again, both peaks of the fundamental harmonic (blue and red) have an $n=1$ toroidal structure, and a second harmonic peak (green) has an $\mathrm{n}=2$ structure.

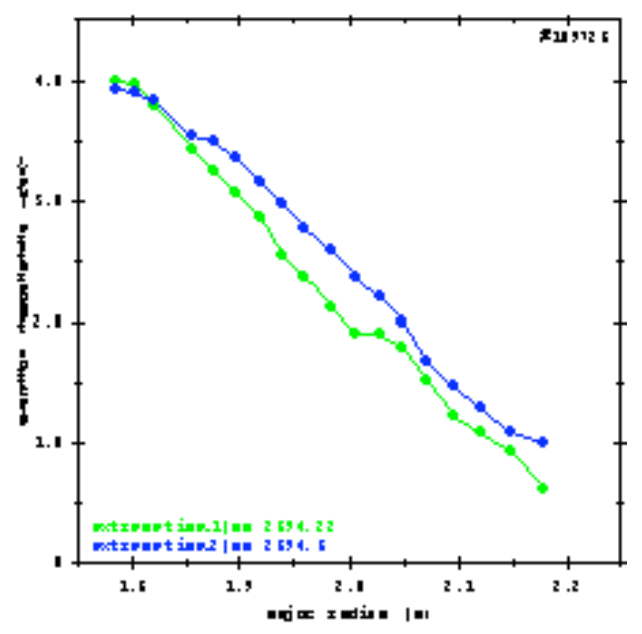

Fig. 16 Radial variations of $T_{e}$ during oscillations are shown (one $T_{e}$ signal shown in Fig. 8). $T_{e}$ profiles at two extreme time phases in a cycle of oscillations show a dominantly global kink-like nature of the perturbations.

These oscillations arise from global kink-like perturbations. Figures 16 and 17 examine features of radial variations of $\mathrm{T}_{\mathrm{e}}$ in these oscillations. Figure 16 shows $T_{e}$ profiles at two time points, corresponding to two extreme phase points in a cycle of oscillations - this pair is from the last full cycle of oscillations before the mode locking. Figure 17 shows the difference ("perturbation $\mathrm{T}_{\mathrm{e}}$ ") between these two profiles. Three components may be recognizable in the perturbation $\mathrm{T}_{\mathrm{e}}$ : a largely negative dominant component extending over most of the plasma cross-section, a negative component confined in the edge region, and a positivelycontributing component from an island, $3-4 \mathrm{~cm}$ wide and centered around $\mathrm{R}=202 \mathrm{~cm}$ (island is more evident in the profile plot in Fig. 16). The oscillations are thus predominantly of a global nature, like perturbations in a kink mode. The perturbation $\mathrm{T}_{\mathrm{e}}$ is large, reaching $0.5 \mathrm{keV}$. The flux surface excursion is up to $6 \mathrm{~cm}(\sim 15 \%$ of the plasma half-width on the outboard side), assuming a constant $T_{e}$ on a flux surface during the oscillations. The localized perturbations associated with the modest-size island do not exhibit a characteristic phase reversal because they are dominated by the kink-like distortions across the radial profile.

The oscillations depicted in Figs. 8 and 13, and analyzed in Figs. 14-17, may be a phenomenon not peculiar to the DIII-D device alone. Perturbation $T_{e}$ profiles consisting of three components that are similar to those in Fig. 17 have also been seen in high $\square$ discharges on the TFTR tokamak [44]: the global component was termed the kink-like mode (KLM), the edge component was called the edge originated magnetic perturbations (EOMPs), and the island component was referred to as the tearing mode (TM). The three components on TFTR appeared in different permutations [Figs. 8-11 and 16-19 in ref. 44]. The EOMP was also observed alone, exhibiting large external magnetic perturbations with no counterpart $T_{e}$ perturbations in the plasma interior - a peculiar situation for MHD modes [Figs. 11 and 19 in ref. 44]. Analysis led to a hypothesis that the EOMP was magnetic field generated by current flowing in the SOL, though TFTR lacked diagnostics to measure it.

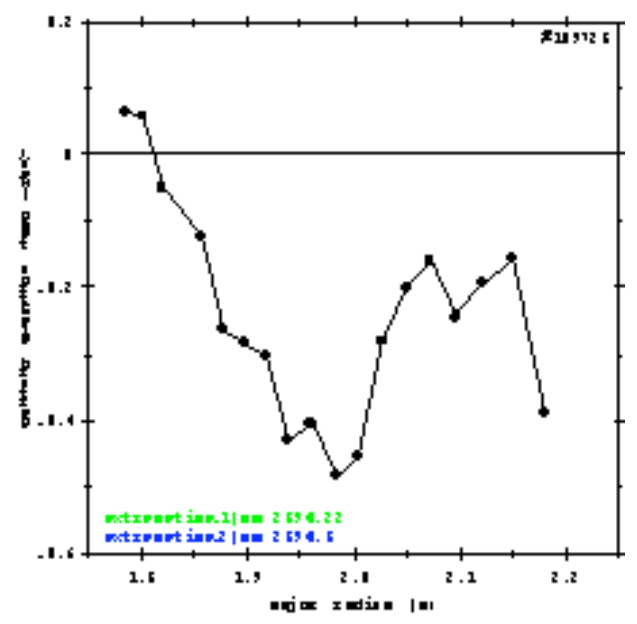

Fig. 17. Difference between the two profiles (perturbation $\boldsymbol{T}_{e}$ ) shown in the previous figure is plotted. It consists of a dominant negative component extending over the plasma cross-section, a negative component in the edge region, and a positive component from an island, $3-4 \mathrm{~cm}$ wide and centered around $R=$ $202 \mathrm{~cm}$ (island is more evident in the profile plot in the previous figure).

The appearance of the saturated kink-mode-like oscillations is examined further in the discussion section. 


\subsection{SOLC during fast MHD activity}

Events in the SOLC also occur, closely correlated with magnetic and thermal signatures commonly associated with fast MHD activity. These SOLC events are thus likely to be an integral part of the MHD activity.

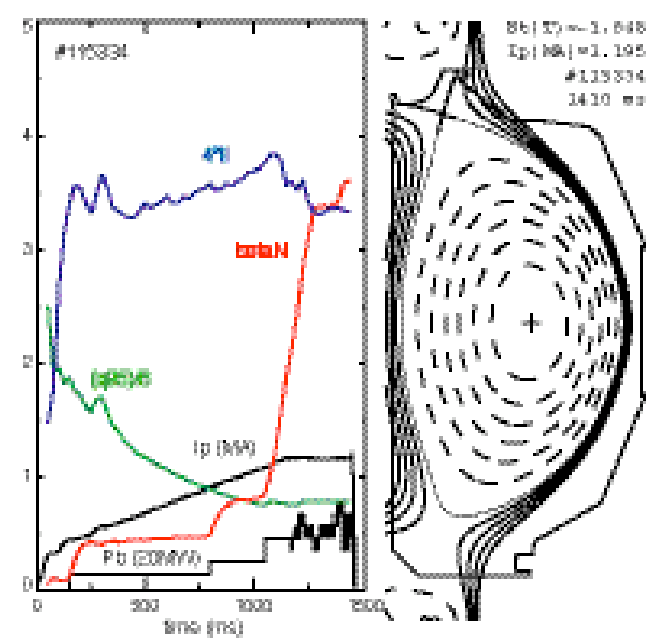

Fig. 18 A pictorial discharge summary for an RWM discharge (see caption to Fig. 3 for explanation). A DN discharge in which $\square_{N}$ attains the no-wall $\square$ limit during an $I_{p}$ flattop period.

Figure 18 shows a pictorial discharge summary for a (slightly upward biased) DN discharge that ended in a disruption. The discharge has a standard DIIID magnetic configuration and field lines with lefthanded helical twist. The evolution of the discharge is briefly described as background information. $\square_{\mathrm{N}}$ exceeded the nominal no-wall limit of $4 l_{i}$ for the $\mathrm{I}_{\mathrm{p}}$ flattop period. The last $\square_{\mathrm{N}}$ value calculated - at the end of the red curve in Fig. 18 was 3.6 at $1425 \mathrm{~ms}$. The discharge disrupted at $1440 \mathrm{~ms}$ (the onset time of the current quench). Mirnov signals exhibited coherent oscillations in the frequency range, $30-70 \mathrm{kHz}$, over a period lasting more than two hundred ms before the disruption. Two MHD events were detected by the Mirnov diagnostic during a brief period of interest leading up to the disruption: the first one at 1431 ms with a peak-to-peak variation of $\sim 20 \mathrm{G}$ (not shown) and a second at $1439 \mathrm{~ms}$ with $\sim 200 \mathrm{G}$ (see Fig. 21, panel (c)). (These events were probably a phenomenon separate from the coherent oscillations, as the oscillations appeared to persist during the onset phase of the MHD events.) The SOLC signals exhibited a characteristic ELMing behavior similar to the one shown in Figs. 5 and 6, with the last ELM occurring at $1418 \mathrm{~ms}$. Oscillations were not evident in the SOLC signals that corresponded to the coherent magnetic oscillations.

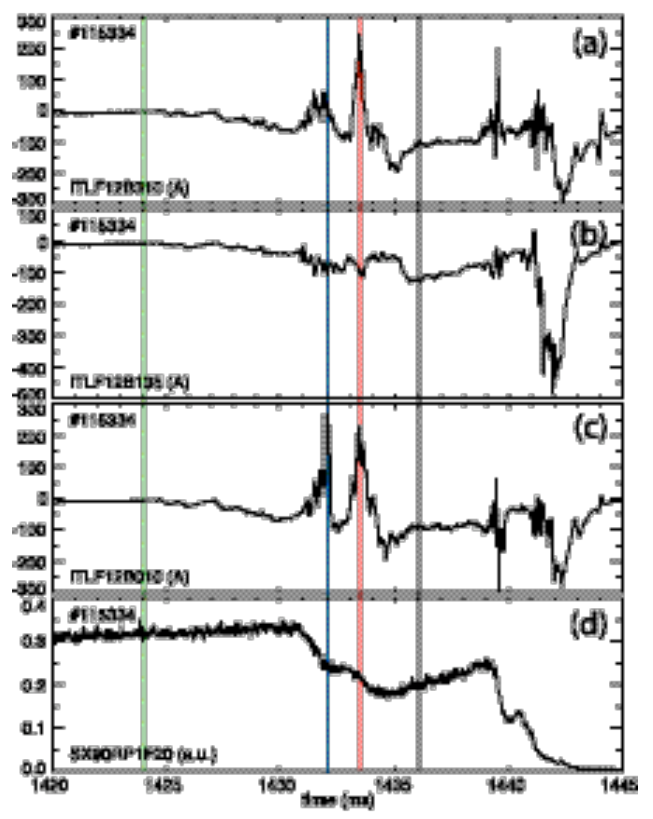

Fig. 19 Shown are three tile current sensor signals from the tile ring \#12B $(a-c)$ and a central SXR signal $(d)$ in the discharge shown in the previous figure. Vertical lines indicate the times for constructing a toroidal variation (next figure).

Figure 19 shows signals from three tile current sensors at different toroidal locations in the tile ring \#12B in the bottom divertor (panels (a) through (c)) and a central SXR signal (panel (d)) over a $25 \mathrm{~ms}$ period that included the disruption. Central SXR signals (one shown in Fig. 19) indicated that the thermal collapse occurred in two stages, with a partial recovery in-between. The first slower collapse began at about $1431 \mathrm{~ms}$, coincident with the first MHD event. Edge SXR emission (not shown), however, began to decrease somewhat earlier at $1425 \mathrm{~ms}$. A second faster collapse started at about $1439 \mathrm{~ms}$, coincident with the second MHD event. Each collapse period appeared to consist of two minor steps. No ECE data was available for this discharge to provide a localized measurement of the internal perturbations. The 
SOLC magnitude began to increase (in the negative direction) at about $1425 \mathrm{~ms}$ (1 ms after the green vertical line in Fig. 19). Two distinct periods of intense activity followed, which were approximately coincident with the two SXR thermal collapse periods. There was also a quiescent interval in-between, approximately corresponding to the SXR partial recovery period.

During the first thermal collapse period, 1431$1435 \mathrm{~ms}$, the signals in the panels (a) and (c) of Fig. 19 had two prominent spikes (indicated by the blue and red lines). The signal in the panel (b) was quieter in comparison. The SOLC were still large during the quiescent period, 1436-1439 ms (beginning at the black line), compared to values before the MHD activity, but they were similar in size to each other.

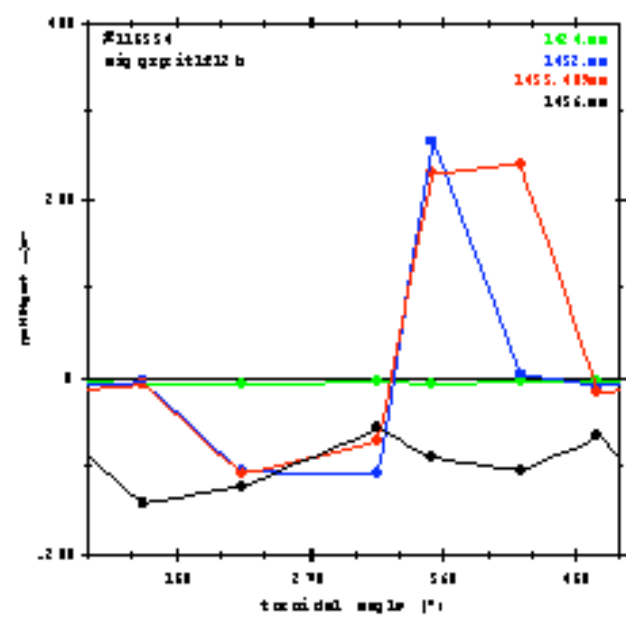

Fig. 20 Shown are the toroidal variation of the SOLC presented in the previous figure at color coordinated time points. The blue and red curves are toroidally nonaxisymmetric and bi-polar. The green and black curves are nearly axisymmetric.

The toroidal variation of the SOLC is depicted in Fig. 20 at four time points indicated by colored vertical lines in Fig. 19. The time points are colorcoordinated between the two figures. All six available signals (three of which were shown in Fig. 19, panels (a)-(c)) were used in constructing these curves. The green curve indicates that the current was small before the onset of the MHD activity. The blue and red profiles are toroidally non-axisymmetric. The blue curve shows significant current only at three of the six measuring points, indicating that the current distribution was narrow or spatially spiky. The red curve may be interpreted as a more global $n=1$ like distribution together with an $n=0$ offset. The blue and red curves have a toroidally bi-polar variation, suggesting that the EMF of the MHD activity may have driven the SOLC during the first thermal collapse period. But the thermoelectric potential cannot be ruled out as an alternative or additional mechanism. The toroidal flux swing may have also contributed. A loss of thermal energy generates a poloidal loop voltage in a direction of maintaining existing diamagnetic current. The SOLC driven by a toroidal flux swing then has, with $B_{t}<0$, a positive sign at the bottom outboard divertor (ring \#12B), and flows, with field lines having a left-handed twist, in a direction opposite from the ohmic plasma current.

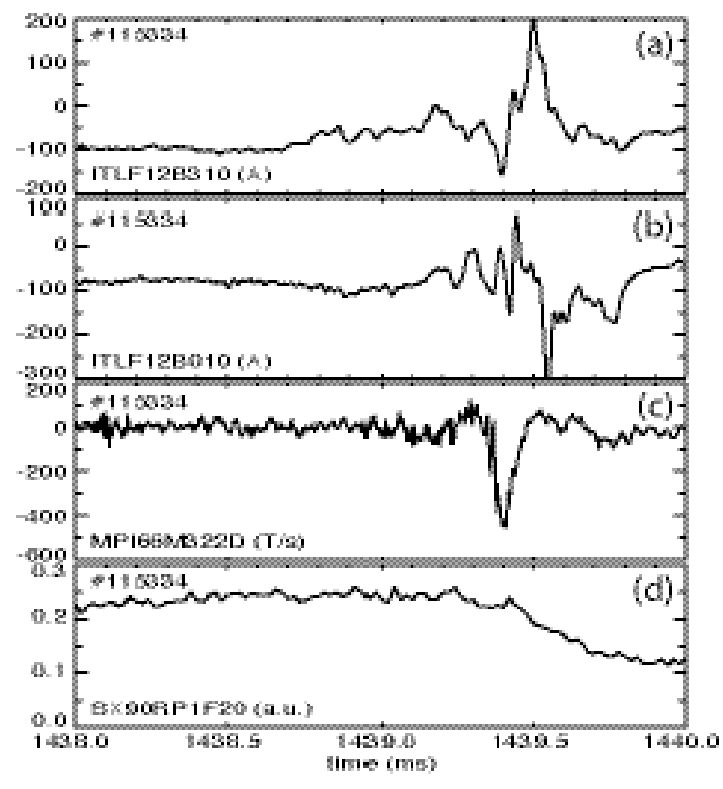

Fig. 21 Second of the MHD events shown in Fig. 19 in an expanded time scale: (a) and (b) tile current signals from the tile ring \#12B, (c) outboard mid-plane Mirnov signal, and (d) central SXR signal. The activiy in the SOLC occurs during the same period, around one ms long, as the activity in Mirnov and SXR occurs during the MHD event. The SOLC is not a simple effect of MHD activity, but is rather intergral part of it.

The black curve in Fig. 20 shows that the toroidal variation is much reduced during the period of partial recovery in the SXR signals between the two thermal collapses. 
During the second thermal collapse period, 1439$1444 \mathrm{~ms}$, two features are evident in the signals shown in the panels (a) and (c) of Fig. 19: a sharp spike at $1439.4 \mathrm{~ms}$ and a much broader feature around $1442 \mathrm{~ms}$. The signal in the panel (b) was smaller during the sharp spike, but was greater during the broad feature, in comparison with the signals in the other two panels. The sharp spike is examined on a greatly expanded time scale in Fig. 21. Two of the three SOLC signals and the SXR signal shown earlier in Fig. 20 are plotted here. The signal from a Mirnov coil located on the outboard mid-plane is also shown. Several cycles of growing oscillations suggestive of instability are discernible in the SOLC signals beginning at about $1438.9 \mathrm{~ms}$ The spiky feature peaking at $1439.4 \mathrm{~ms}$ in the Mirnov signal represents variation of magnetic field in excess of $200 \mathrm{G}$ over a period of about $200 \square$ s. The central SXR signal begins to decrease approximately coincident with this spike.

The sharp spike at $1439.4 \mathrm{~ms}$ in the SOLC signal in Fig. 19 is toroidally bi-polar (as evident from the plot of the same feature on an expanded scale in Fig. 21), suggesting that it may be mainly driven by the EMF of the MHD activity, and, though much faster, is of the same nature as the event during the first thermal collapse period. In contrast, the broad feature at $1442 \mathrm{~ms}$ in Fig. 19 exhibited a uni-polar toroidal variation. As field lines had a left-handed twist and the current flowed out (negative SOLC signals) of the bottom divertor plates (ring \#12B), the SOLC was in the same toroidal direction as the ohmic plasma current during the broad feature. Also, the plasma current quench process was already in progress by this time. Considering these factors, it was likely that the broad feature in the SOLC was driven by the poloidal flux swing of the plasma column, and thus was a phenomenon similar to the one described earlier in Fig. 10.

\section{Radial Profile of SOLC}

The radial profile of the SOLC is an important piece of information needed in assessing the phase mixing effect as well as in constructing the circuit topology. But it is often difficult to determine a radial profile during MHD activity because of its rapid time variation. Instead, a radial profile is determined here, in a quiescent discharge described earlier in Sec. 3.3, using a "swept strike point method."

\subsection{Swept Strike Point Method}

The tile current sensor does not have the spatial resolution necessary to determine a radial current density profile because it integrates current density over a radial range comparable to the profile width. A swept strike point method may be used to overcome this limitation [5]. The strike point is moved in such a way to "sweep" a radial profile of SOLC over the edge of a tile ring (boundary between two adjacent rings), thereby varying with time the radial range of integration. The sensor output may be calculated using a trial radial profile function, and the time variation of the calculated output may be compared with the measurement to obtain a best-fit profile. The assumptions underlying the swept strike point method are: (a) discharge conditions remain steady during the strike point motion, and (b) SOLC radial profile is a time-invariant function only of the distance relative to the strike point.

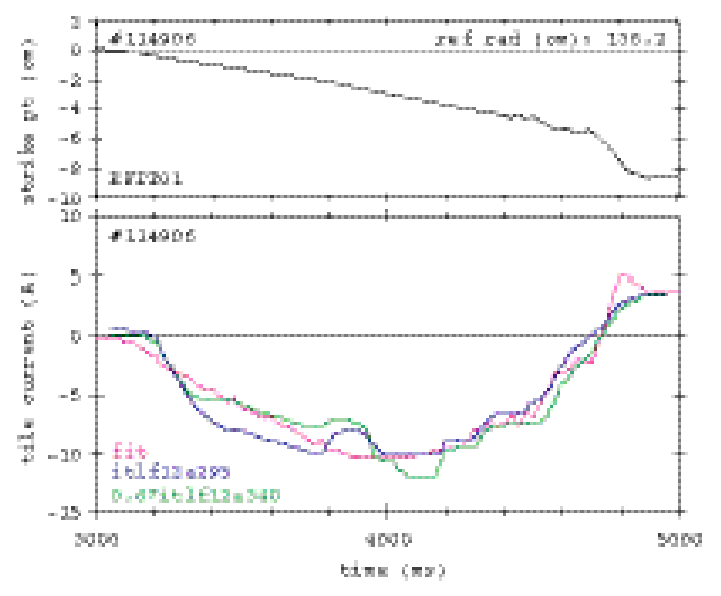

Figure 22. (Top) time variation of the top outboard separatrix strike point location, which moves from a fully "closed gap" position to a nearly fully "open gap" position; (bottom) time variations of SOLCs measured in the latter half of the discharge shown in Fig. 4 after binning and smoothing, with the $340^{\circ}$ sensor signal (green) normalized to the $295^{\circ}$ sensor signal (blue). Calculated values (magenta) use the radial profile trial function shown in Fig. 24. 
Figure 22 (top panel) describes the time variation of the top outboard strike point location, relative to an arbitrarily selected reference radius (see below), obtained in equilibrium calculations using the EFIT code for a late period in the discharge shown in Fig. 3. The strike point moves by $8.6 \mathrm{~cm}$ in 1700 ms from $3200 \mathrm{~ms}$ till $4900 \mathrm{~ms}$, nearly linearly, except for the last $200 \mathrm{~ms}$. Estimated uncertainty in the strike point location is about $8 \mathrm{~mm}$. The flux surface expansion ratio from the outboard midplane to the top outboard divertor plates (ring \#12A) for this discharge is about 5.7 for surfaces within the first $0.5 \mathrm{~cm}$ of the separatrix as measured in the outboard mid-plane, and falls off to about 4.3 at $2 \mathrm{~cm}$.

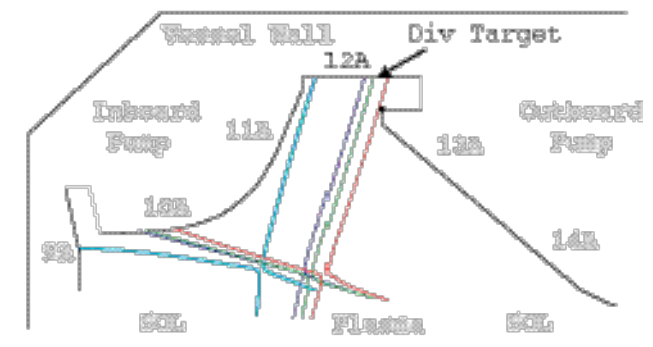

Fig. 23 The top divertor region is sketched schematically (compare with center inset in Fig. 1). The outlines of plasma-facing tile surfaces and vacuum vessel are shown in black curves, together with separatrices in colored curves at four time points: 3200 ms (red), $3725 \mathrm{~ms}$ (green), $4000 \mathrm{~ms}$ (blue), and $5000 \mathrm{~ms}$ (cyan). The gap leading to the divertor is nearly fully closed at $3200 \mathrm{~ms}$ and nearly fully open at $5000 \mathrm{~ms}$. Geometry at $3725 \mathrm{~ms}$ is similar to that at $2000 \mathrm{~ms}$ during the QHM period, with gap opening of $\sim 2 \mathrm{~cm}$.

Figure 23 depicts schematically the separatrix geometry in the top divertor region for this discharge (compare with center inset in Fig. 1). The outlines of plasma-facing surfaces of tiles and vacuum vessel wall are shown in black curves, and the separatrix geometry is shown in colored curves at four time points. When the strike point is at a sufficiently large radius (at and beyond the red curve), the divertor target plates (ring \#12A) are either in the private flux (PFX) region, or in the "shadow" of the protruding edge (black dot) of the neighboring baffle plates (ring \#13). The "gap" leading to the divertor plates, between the separatrix and the baffle plates' protruding edge, is closed. This will be referred to as a fully "closed gap" configuration in this article. The strike point $(138.2 \mathrm{~cm})$ under these conditions was taken as the reference radius in the previous figure. A fully "open gap" configuration results when the strike point is at the inboard edge $(128 \mathrm{~cm})$ of the divertor plates.

\subsection{Measured radial profile of SOLC}

The measured SOLC data shown in Fig. 4 contain spikes and oscillations, especially outside the QHM period (1550-3800 ms). A "DC component" is extracted from the data through smoothing, which are for the most part sampled at $200 \mathrm{kHz}$. The data is first binned and averaged (200 time points) and then smoothed ("repeated moving mean" method using 101 binned points) in order to obtain variations on the time scale of the strike point sweep. The smoothed data are shown in Fig. 22 (bottom panel). The $340^{\circ}$ sensor signal (green) is multiplied by 0.6 to normalize it to the $295^{\circ}$ sensor signal (blue).

Only gross characteristics of the radial profile need to be extracted from the smoothed curves shown in Fig. 22, as details are likely to vary from one discharge to another. Some characteristics are immediately evident from simple inspection of the curves. Negligible (integrated) current flows deep inside PFX region, as the signals are nearly zero when the gap is fully closed. The measured amplitude goes through a maximum (in a negative domain), instead of asymptotically reaching a steady value, implying that the current density reverses its polarity as a function of radial distance. The current begins to rise immediately after the gap opens, implying a finite current density at the strike point. The measured amplitude also ends in a domain (sign) opposite from the initial domain, implying that the reversed polarity lobe of the current density profile is greater than the initial polarity lobe.

A trial radial current density profile function consisting of a cosine function with exponentially varying amplitude is constructed to satisfy these characteristics: $A \exp \left(-\mathrm{x} / \mathrm{L}_{\mathrm{s}}\right) \cos \left(2 \square \mathrm{x} / \mathrm{L}_{\mathrm{w}}+\square\right)$. Here $\mathrm{A}$ is the current density amplitude, $\mathrm{x}$ is the distance measured away from the separatrix (into the SOL), and $\mathrm{L}_{\mathrm{s}}, \mathrm{L}_{\mathrm{w}}$, and $\square$ are fitting parameters. The 
current density inside the PFX region, in the immediate vicinity of the strike point, was modeled by a separate exponentially decaying function: $\exp \left(-\mathrm{x} / \mathrm{L}_{\mathrm{P}}\right)$, where $\mathrm{x}$ is also the distance measured away from the strike point (into the PFX region) and $\mathrm{L}_{\mathrm{P}}$ is a fitting parameter (but usually taken as a small fixed value). The calculated tile current (magenta) and the measured variations (green and blue) are compared over the entire time range shown in Fig. 22 (bottom panel) to accomplish fitting. The fitting parameters are $\mathrm{A}=4.7 \mathrm{~A} / \mathrm{cm}$, $\mathrm{L}_{\mathrm{s}}=-60 \mathrm{~cm}$ (very slowly growing amplitude), $\mathrm{L}_{\mathrm{w}}=$ $9.5 \mathrm{~cm}, \square=0.875 \square$. Figure 24 shows the calculated current density radial profiles at four time points as a function of the major radius. An arrow indicates the strike point location at each time point. The "area under the curve" is the current that would be "measured" by a tile current sensor, and obtained by integrating the curve from the left edge of the plot $(128 \mathrm{~cm})$ to the right-hand side integration limit $(138.2 \mathrm{~cm})$ indicated by a gray vertical line segment.

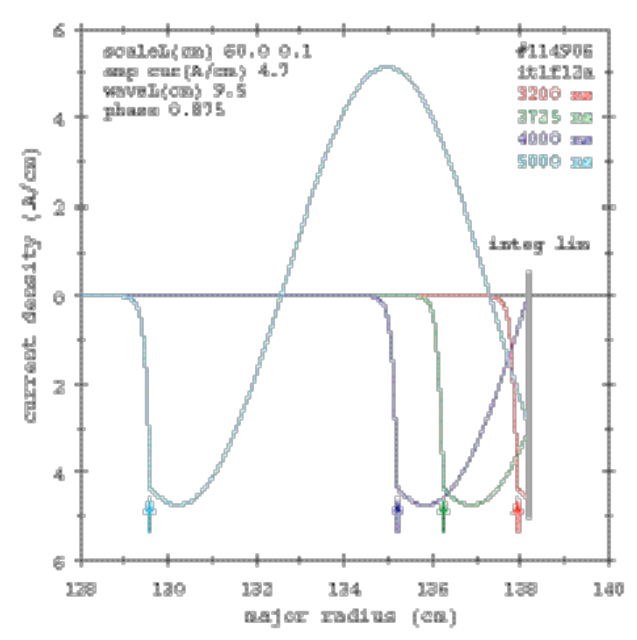

Figure 24. A trial radial profile function for the SOLC in the top outboard divertor is depicted as a function of the major radius. "Integral under the curve" yields current measured by a tile current sensor on the ring \#12A. Integration limits are the left edge of the plot and the "integ limit" indicated by a gray vertical bar.

The curve at $5000 \mathrm{~ms}$ (cyan) in Fig. 24 for the almost fully "open gap" configuration reveals the nearly entire radial profile. The strike point is at $\mathrm{R}=129.6 \mathrm{~cm}$. The current density reverses its polarity about every $5 \mathrm{~cm}$, reflecting the "wavelength" $(9.5 \mathrm{~cm})$ of the underlying cosine function. The first reversal occurs at $\mathrm{R}=132.5 \mathrm{~cm}$ ( $2.9 \mathrm{~cm}$ away from the strike point as measured along the tile surface), and again at $137.3 \mathrm{~cm}$ (7.7 cm away). The positive "lobe" of the profile peaks at about $135 \mathrm{~cm}$ (5.4 cm away). Using the flux expansion factor obtained earlier these polarity reversal points correspond approximately to $0.5 \mathrm{~cm}$ and $1.5 \mathrm{~cm}$ respectively when mapped to the outboard mid-plane. The positive lobe peak is at $1.0 \mathrm{~cm}$, and the reference point $(138.2 \mathrm{~cm})$ maps to $1.7 \mathrm{~cm}$, both in the mid-plane. The negative lobe of the current density profile thus corresponds approximately to the strong phase mixing region, and the positive lobe falls into the weak phase mixing region. These inferences were reached by disregarding the difference in the configuration between the present case and one in Fig. 22. Closely spaced forward and return current paths would generally result in cancellation of field generated by them. But the different efficacy of current in the two paths (here the negative and positive lobes) in generating error field may make the field cancellation less complete.

Radial profiles at other time points are obtained by simply translating the fully open gap profile (cyan) to larger major radius points, and truncating it at the integration limit (gray vertical line). The curve for $3200 \mathrm{~ms}$ (red) is for a nearly fully closed gap configuration. The curve for $4000 \mathrm{~ms}$ (blue) captures the entire negative lobe of the profile, and corresponds to the peak (in negative domain) of the measured current (see Fig. 4). The curve for 3725 ms (green) captures $85 \%$ of the calculated negative lobe although the gap opening at this time is only about $2 \mathrm{~cm}$ as measured from the protruding point of the adjacent baffle plate (black dot in Fig. 23). The separatrix geometry at this time is similar to that at $2000 \mathrm{~ms}$ during the QHM period discussed in Sec. 3.1. The current measured at that time is also about $85 \%$ of the peak negative value ("DC" component) measured over the entire discharge, and is consistent with the calculations discussed here. The observed amplitude of oscillations in the SOLC at the EHO frequency is the result of collecting majority $(\sim 85 \%)$ of the negative current lobe, but none of the positive lobe. EHO oscillations may actually involve larger current than has been measured, which is located farther out in the positive lobe region. 


\subsection{Return Current Path}

The existence of polarity reversal, as a function of radial location, might appear as a surprise. But it has also been observed under other circumstances in DIII-D. First, the current at the peak of the ELM in a LSN discharge has been observed [9] to reverse its polarity as the strike point was swept past the tile ring boundary, as has been done in the present work. Second, time-resolved measurements during the ELM in a DN discharge have shown [9], without having recourse to a swept strike point method, that the SOLC spreads over a large radial distance, spanning three tile rings in the bottom divertor, and reverses its polarity between two adjacent rings. The current at a ring farther away from the strike point was sometimes greater than the current at a ring closer to the strike point.

A bi-polar current in a narrow region at or near the separatrix strike point has been identified [3] in JET as being driven by the Pfirsch-Schlüter effect in the SOL. The total width of the negative lobe of this bi-polar feature was about $2 \mathrm{~mm}$, when mapped to the outboard mid-plane. A bi-polar current with a radial structure similar to that observed in JET has also been seen earlier in DIIID [4] and more recently in TCV [13]. The current direction in the bi-polar distribution in Fig. 24 is consistent with an interpretation in terms of the Pfirsch-Schlüter effect. But the width of the negative lobe is about $5 \mathrm{~mm}$, when mapped to the outboard mid-plane, significantly wider than the feature found in JET. But the comparison is between two different devices. The analysis method employed here is also not suitable in identifying radially narrow features. More work is needed before reaching a definitive interpretation of the observed bi-polar radial profile shown in Fig. 24.

The reverse current path may represent a return current path within the SOL itself. This is in addition to, or in place of, postulated return path through the tokamak structure. The presence of a return path within the SOL would complicate calculations of error field generated by an SOLC. This may also be another reason why a "total quantitative accounting" of the SOLC is difficult as discussed in Sec. 3.2.

\section{Conclusion}

1. Currents have been observed to flow during MHD activity in the scrape-off layer (SOL) of poloidally diverted $\mathrm{H}$-mode discharges in the DIII-D tokamak heated by neutral beam injection (NBI) to high $\square_{\mathrm{N}}$ regimes, including:

a. SOLCs, slowly growing with a several $\mathrm{ms}$ time scale, during a locked-mode-like sequence of events characterized by data from magnetic and $\mathrm{T}_{\mathrm{e}}$ diagnostics and toroidal rotation measurement.

b. SOLCs, oscillating in the frequency range of several $\mathrm{kHz}$, phase-locked with magnetic and $T_{e}$ perturbations at the same frequency, and having substantially identical spectral characteristics and a torodial structure as those of magnetic perturbations.

c. SOLCs, rapidly growing with a sub-ms time scale, correlated with magnetic and thermal signatures of a thermal collapse and a current quench.

d. SOLCs, spiky in temporal behavior, correlated with spiky features in $\mathrm{D}_{\square}$ signals commonly identified with the ELM.

These currents flow in close correlation with other discharge parameters commonly used to characterize MHD activity, and may be an integral part of the MHD activity under observation, rather than its merely inconsequential effect.

2. SOLCs observed during MHD activity are characterized by:

a. The propensity to flow in a toroidally nonaxisymmetric pattern, even though underlying driving mechanisms may inherently be axisymmetric, thus serving as a flux converter.

b. The magnitude of "total current involved" comparable to external coil currents that are believed capable of influencing the MHD stability. 
3. Candidate mechanisms for driving the SOLC during MHD activity have been identified:

a. Thermoelectric potential that is toroidally non-axisymmetric.

b. Flux swing, both toroidal and poloidal, induced by the loss of thermal energy and changes in plasma current, which produces a uni-polar toroidal distribution.

c. Electromotive force (EMF) of MHD activity, which produces a bi-polar toroidal distribution.

However, how a toroidally non-axisymmetric thermoelectric potential distribution would come about during MHD activity is presently not known.

4. With the above observations (1 and 2) and possible driving mechanisms (3) taken together, the SOLC has the potential to play a role in the MHD stability physics, stabilizing, if purely reactive, or possibly destabilizing, if not purely reactive. The influence, even though stabilizing, may still be important, just as stabilizing image currents in the vessel wall play a crucial role in the external kink stability.

5. An effect has been identified, which stems from the shear in the field line pitch angle, and mixes the toroidal phase of field lines distributed over a finite radial zone. In an LSN discharge, a radial region, about $0.4 \mathrm{~cm}$ wide, of strong phase mixing was found to lie immediately adjacent $(0-0.4 \mathrm{~cm})$ to the separatrix, and a much broader region, about $1.8 \mathrm{~cm}$ wide, of weak phase mixing was seen to lie farther out $(0.4-2.2 \mathrm{~cm})$ in the SOL, with all quoted distances mapped to the outboard mid-plane; this "phase mixing effect" tends to mitigate the ability of a non-axisymmetric SOLC to generate a non-axisymmetric error field.

6. Characteristics of the SOLC, needed for quantitatively assessing its magnetic consequences, have been identified: (a) current intensity, (b) current density distribution, both toroidal (non-axisymmetry) and radial (phase mixing), and (c) current circuit topology, including return current path (field cancellation) and circuit geometry (inductive coupling).

Whether the SOLC actually played a role in the MHD stability under a specific set of conditions depends importantly upon its actual circuit topology and radial distribution; these properties are difficult to measure during MHD activity, and have not yet been determined.

7. The SOLC possesses the potential for other magnetic consequences: (a) its error field may introduce complications to feedback control schemes that detects a mode pattern and counters it with field produced by external coil current because dynamic error field generated by the SOLC does not in general mirror the spatial pattern of MHD modes inside the plasma, and (b) its non-axisymmetric field detected by toroidally discrete magnetic sensors may be falsely interpreted as axisymmetric field by the tokamak control logic and in equilibrium reconstruction.

8. A radial profile of SOLCs observed during a quiescent discharge period in a USN discharge has been determined, using the tile current diagnostic with a swept strike point method; it possesses polarity reversals as a function of radial distance, with a first lobe approximately corresponding to a region of strong phase mixing, and a second lying in a region of weak phase mixing.

\section{Discussion}

Discussion is offered here, based on experimental observations mixed with conjecture, in order to expound more freely on the implications of the conclusions drawn, stimulate new ideas, and indicate a direction for future effort.

\section{- Causality Issues}

It is sometimes argued that the SOLC is an effect of MHD activity, often with an implied assertion that it is therefore inconsequential. Regardless of the veracity of the argument on the SOLC's origin, 
however, the conclusion that it is inconsequential does not necessarily follow. Just as image current in the vessel wall, which is an effect of MHD activity, plays an indispensable role in determining the stability of some MHD activity, such as the RWM, the SOLC may also play a role in the evolution of MHD activity, including its stability. Possessing driving mechanisms independent of MHD activity, the SOLC may have destabilizing as well as stabilizing influence.

Time-dependent simulation [45] of a feedback control stabilization scheme for the RWM indicates that it takes a period of a few ms before the growth of the instability can be arrested. Such analysis typically assumes that the system to be controlled, consisting of the plasma, the vessel wall, and a vacuum region in-between, does not change during that period ("linear" analysis). But measurements reported here demonstrated that the SOLC can rise to significant magnitude in a sub$\mathrm{ms}$ time scale. Thus, a new element intrudes into the system while the feedback control is at work to arrest the instability. The SOLC can thus "nonlinearly" influence the performance of the feedback control. Now that this work has demonstrated that the SOLC is an integral part of the MHD activity itself, the central issue is whether or not the SOLC has quantitatively important consequences in MHD activity.

Advancing beyond a qualitative assessment of SOLC's role in the stability physics, provided in this work by comparing "total current involved" with coil currents used in feedback stabilization, will require significant experimental and theoretical effort. First, a complete description of current paths must be experimentally established. The task is a lot more difficult to accomplish during fast MHD activity because measuring instruments often lack either the necessary time response or spatial resolution. Second, the inclusion of the SOLC in stability analysis would require three-dimensional MHD codes, which are just beginning to become available for the tokamak. Some codes may need extension in order to treat the SOLC, which is not an ideal MHD element.

Definitive experimental assessment of the influence of the SOLC may be conducted by either cutting off, or greatly reducing, the SOLC. Reduction of the halo current by an order of magnitude by increasing the halo current circuit resistance has recently been demonstrated in a VDE experiment in MAST [27]. Enforcing toroidal axisymmetry of the SOLC by feedback controlled external power supplies also holds promise as a step toward active exploitation of the SOLC for controlling MHD activity. The measurement reported in the present work indicates that merely applying a biasing voltage to a toroidally contiguous conducting ring is likely to result in a toroidally non-axisymmetric SOLC distribution. It is instead proposed here to enforce axisymmetry directly upon the current in order to overcome the demonstrated SOLC's propensity to flow in a non-axisymmetric pattern whether or not the driving mechanism is inherently axisymmetric. This proposal is similar to the "segmented divertor biasing experiment" described in ref. [30].

\section{Dynamic Error Field}

The magnetic field produced by the SOLC is an error field, just like the field generated by structural defects of the tokamak, in that it is toroidally non-axisymmetric. But the SOLC comes and goes, depending on the discharge conditions, and is thus a dynamic source of error field, in contrast to a structural error field that is permanently present and static by nature. The error field produced by the SOLC may be a better candidate for explaining phenomena conventionally attributed to structural error fields, such as mode slow-down and locking, when they occur in some discharges but not in others. The magnitude of the error field generated by the SOLC, evaluated based on a model sheet current of infinitesimal thickness [46], can be a significant fraction of a component of the equilibrium field, especially in divertor regions.

\section{Driving Mechanisms}

The electromotive force (EMF), generated by the time-varying magnetic field of MHD activity and acting on a SOLC circuit, may be a natural candidate for driving the SOLC. It may be calculable using existing analysis codes. However, no such calculations appear to be available at present, to the best of authors' knowledge. On the 
one hand, large-amplitude oscillating SOLCs examined in Sec. 5.2 may be a prime candidate for current driven by the EMF of MHD activity. On the other hand, growing quasi-stationary SOLCs observed, following mode locking in the same discharge, and discussed in Sec. 5.1, may serve as a prime example of current that is demonstrably not driven by the EMF of MHD activity.

A semi-quantitative analysis is offered here for the growing quasi-static SOLCs shown in Fig. 13.

Postulate that the quasi-static SOLCs were driven by the EMF, and examine whether or not contradictions result. Analysis is based on a simple coupled circuit model in which the MHD activity is represented by an effective current. The equation governing the SOLC may be written as $d / d t\left(\mathrm{I}_{\mathrm{SOL}}\right)$ $+\left(\mathrm{R}_{\mathrm{SOL}} / \mathrm{L}_{\mathrm{SOL}}\right) \mathrm{I}_{\mathrm{SOL}}=\left(\mathrm{MC}_{\mathrm{MHD}} / \mathrm{L}_{\mathrm{SOL}}\right) d / d t\left(\mathrm{~B}_{\mathrm{MHD}}\right)$, where $I_{S O L}$ is the current, $R_{S O L}$ is the resistance, and $\mathrm{L}_{\mathrm{SOL}}$ is the self-inductance, all of the SOLC circuit, $\mathrm{B}_{\mathrm{MHD}}$ is magnetic field, $\mathrm{C}_{\mathrm{MHD}}$ is a conversion factor between magnetic field and effective current, both of MHD activity, and M is the mutual inductance between the SOLC and the MHD current circuits.

The $\mathrm{L} / \mathrm{R}$ time of the SOLC circuit is estimated to be of order $\mathrm{L}_{\mathrm{SOL}} / \mathrm{R}_{\mathrm{SOL}} \sim 0.2 \mathrm{~ms}$. The quantity inside the parenthesis, (..), on the right-hand-side of the governing equation can be determined from the measured values of $\mathrm{I}_{\mathrm{SOL}}$ and $d / d t\left(\mathrm{~B}_{\mathrm{MHD}}\right)$. Because the angular frequency, $\square=2 \square \times 6 \mathrm{kHz}=3.8 \mathrm{x}$ $10^{4} / \mathrm{sec}$, (over the time interval $2578-2580 \mathrm{~ms}$ ) is much greater than the reciprocal L/R time, $5 \mathrm{x}$ $10^{3} / \mathrm{sec}$, the inductive term dominates over the resistive term on the left-hand-side of the equation during the oscillating period, and hence $(\ldots) \approx \square \mathrm{I} /$ $d / d t\left(\mathrm{~B}_{\mathrm{MHD}}\right)=3.8 \times 10^{4} / \mathrm{sec} \times 80 \mathrm{~A} / 270 \mathrm{~T} / \mathrm{sec}=$ $1.1 \times 10^{4} \mathrm{~A} / \mathrm{T}$. The resistive term dominates over the inductive term after mode locking. In order to be consistent with the measured peak SOLC, $d / d t$ $\left(\mathrm{B}_{\mathrm{MHD}}\right)=\left(\mathrm{R}_{\mathrm{SOL}} / \mathrm{L}_{\mathrm{SOL}}\right) \mathrm{I}_{\mathrm{SOL}} /(\ldots)=270 \mathrm{~A} /(0.2 \mathrm{~ms} \mathrm{x}$ $\left.1.1 \times 10^{4} \mathrm{~A} / \mathrm{T}\right) \sim 120 \mathrm{~T} / \mathrm{sec}$. This value is inconsistent with the observed value, $\sim 3 \mathrm{~T} / \mathrm{sec}$, after mode locking (Fig. 13, panel (e)) by a factor of $\sim 40$. Thus, it is unlikely that the quasi-static SOLC observed in this discharge was driven directly by the EMF of the observed MHD activity.

Some estimates may be given for the voltage generated by the flux swing of the plasma column.
A thermal collapse may typically begin with the edge $\mathrm{T}_{\mathrm{e}}$ "drooping" on a few ms time scale, or undergoing multi-step, small-scale crashes, and end with central $\mathrm{T}_{\mathrm{e}}$ crashing on a sub-ms time scale, thus losing most of electron thermal energy, which may account for about a third of the total energy. The toroidal flux in a high beta discharge may be on the order of $100 \mathrm{mWb}$. Thus, a hypothetical $30 \%$ drop in total thermal energy in $0.3 \mathrm{~ms}$ would produce a poloidal loop voltage of $100 \mathrm{~V}$ during the main crash. The toroidal loop voltage generated in current quench is enhanced by the transformer effect - a SOLC path executes typically several toroidal revolutions, nearly seven in the case shown in Fig. 11. With an estimated self-inductance, $\sim 3 \square \mathrm{H}$, of the DIII-D plasma column, a hypothetical $\sim 33 \mathrm{kA} / \mathrm{ms}$ change in the plasma current would produce a toroidal loop voltage of $100 \mathrm{~V}$, which would further be enhanced by the transformer ratio. A very large SOLC could be driven in a current quench process, although presumably the cold SOL plasma after the thermal collapse would require a high loop voltage to drive the current.

\section{Saturated kink-mode-like oscillations}

The appearance of saturated kink-mode-like oscillations shown in Fig. 8 is of interest in two respects. First, the external kink mode is conventionally believed to grow without reaching a saturated state; once unstable, it is thought to lead all the way to a process that removes the source of the instability, such as a thermal collapse or disruption. Second, these oscillations occur in a regime around or above the nominal no-wall limit for kink modes. But they were not the RWM, as their frequency was two orders of magnitude higher than a typical RWM frequency, which is primarily determined by the characteristic time for poloidal field perturbations to penetrate the resistive vessel wall, about $5 \mathrm{~ms}$ for DIII-D. The NTM in conventional theory cannot explain the dominant global kink-like part of the observed oscillations. The width of the observed island, 3-4 $\mathrm{cm}$, is also significantly smaller than the size of an NTM island, $\sim 8 \mathrm{~cm}$, predicted by calculations based on the modified Rutherford equation. It is an interesting question to pose whether or not inclusion of the SOLC as an integral part of MHD 
activity would alter predictions of conventional theory and account for the existence of saturated kink-mode-like oscillations or smaller-thanpredicted NTM islands.

These oscillating perturbations may be an example where the SOLC is possibly driven by the EMF of MHD modes inside the plasma. In that case, the SOLC resembles the image currents in a stabilizing wall, and thus should be stabilizing, and might provide a saturation mechanism for the external kink instability. Conversely, the oscillating SOLC could also be driven by thermoelectric potential. In that case, the relative phase between the oscillating SOLC and the modes in the plasma would be determined by complicated heat transport physics, which is not understood at present. The SOLC could be either stabilizing or de-stabilizing.

\section{Instability in SOL}

Main finding in this work was that the SOLC has the propensity to flow in toroidally nonaxisymmetric pattern during all types of MHD activity examined, regardless of the driving mechanisms involved. The mechanism for creating this tendency is presently not understood. But it is interesting to speculate as to: (a) whether or not an initially axisymmetric SOLC can become itself unstable, and (b) whether its resulting nonaxisymmetric error field can trigger MHD instability inside the plasma, reversing the conventionally believed direction of the causality. No theoretical analysis appears available at present that is directly applicable to the stability of a SOLC layer. It may, however, be noted that the SOLC can be represented as a sheet current of either a finite or infinitesimal thickness and linetied at two end plates. The geometry is not far from that of a straight cylinder in the case of DN discharges (field lines in the SOL of a DN discharge, e.g., see Fig. 18, would be similar to the one indicated by the cyan curve in the right panel of Fig. 11). An experimental effort [47] is underway, though under unrelated circumstances, to investigate the stability of a current sheet in a cylindrical geometry, sometimes referred to as "line-tied screw pinch." The magnetic geometry differs somewhat, however, in that the relative strengths of axial and azimuthal fields in this experiment are opposite from those of the geometrically corresponding vertical and toroidal fields in the tokamak. The outcome of the experiment is nevertheless of great interest.

\section{Diagnostic Needs}

The existence of strong toroidal non-axisymmetry points to the need for more complete coverage in toroidal measurement of the SOLC. Diagnostic tools and methods are needed for determining the circuit topology and radial profile of the SOLC during MHD activity. For this purpose, the present tile current monitors have the needed time response, but not the spatial resolution. A set of more densely arranged tile current monitors would be useful. Langmuir probes have the needed spatial resolution, but not the time response, when operated in a standard way with a biasing power supply. An array of closely spaced Langmuir probe tips, each connected to ground only through a sensing resistor, may combine the advantages of tile current monitors and Langmuir probes.

\section{Acknowledgement}

The authors gratefully acknowledge their indebtedness to the research, technical, and computing crews for operation of the DIII-D tokamak and data acquisition, and to diagnosticians for providing valuable data. This work was supported by U.S. Department of Energy under Contract Nos. DE-AC02-76CH03073, DEAC03-99ER54463, DE-FG03-97ER54415 and DE-AC04-94AL85000.

\section{References}

[1] HARBOUR, P.J., et al., J. Nucl. Mater. 162164(1989)236.

[2] CHANKIN, A.V., et al., J. Nucl. Mater. 196198(1992) 739 .

[3] SCHAFFER, M.J., et al., Nucl. Fusion 37(1997)83.

[4] BUCHENAUER, D., et al., J. Nucl. Mater. 176-177(1990)528.

[5] SCHAFFER, M.J. and LEIKIND, B.J., Nucl. Fusion 31(1991)1750. 
[6] EVANS, T.E., et al, J. Nucl. Mater. 220222(1995)235.

[7] TAKAHASHI, H., et al., 30th EPS Conf. on Contr. Fusion and Plasma Phys., St. Petersburg, 2003, ECA Vol. 27A, P-3.99

[8] FENSTERMACHER, M., et al., Plasma Phys. and Cont. Fusion 45(2003)1597.

[9] TAKAHASHI, H., et al., Bull. Am. Phys. Soc. 48(2003)185.

[10] ITAMI, K., et al., Proc. 14th Int. Conf. On Plasma Phys. and Contr. Nucl. Fusion Research (Wuerzburg) 1992, IAEA-CN-56/A-6-5.

[11] KUMAGAI, A., et al., Plasma Phys. Control. Fusion 39(1997)1189.

[12] NAGASHIMA, K., SHOJI, T., MIURA, Y., Nucl. Fusion 36(1996)335.

[13] PITTS, R.A., et al., Nucl. Fusion

43(2003)1145.

[14] HARBOUR, P.J., in Contr. Plasma Phys.

28(1988) 415

[15] STAEBLER, G.M., Hinton, F.L., Nucl.

Fusion 29(1989)1820.

[16] STANGEBY, P.C., Nucl. Fusion

30(1990)1153.

[17] TSUI, H.Y.W., Nucl. Fusion 28(1988)1543.

[18] THOMAS, P., et al., in Plasma Physics and Controlled Nuclear Fusion Research 1984 (Proc. $10^{\text {th }}$ Int. Conf. London, 1984), Vol. 1, IAEA, Vienna (1985) 353.

[19] JENSEN, T.H. and SKINNER, D.G., Phys. Fluids B2 10(1990)2358.

[20] KUGEL, H.W., et al., in Controlled Fusion and Plasma Physics (Proc. 16th Eur. Conf. Venice, 1989) Vol. 13B, Part I, Eur. Phy. Soc. (1989) 199.

[21] STRAIT, E.J., et al., Nucl. Fusion, 31(1991)527.

[22] GRUBER, O., et al., Plasma Phys. Control. Fusion, 35(1993)B191.

[23] GRANETZ, R.S., et al., Nucl. Fusion, 36(1996)545.

[24] CASTLE, G.G., et al., in Plasma Physics and Controlled Nuclear Fusion Research 1996 (Proc.

$16^{\text {th }}$ Int. Conf. Montreal, 1996), Vol. 1, (IAEA, Vienna) 707.

[25] ABE, M., et al., ibid, p. 777.

[26] NEYATANI, et al., Nucl. Fusion,

39(1999)559.

[27] COUNSELL, G.F., et al., Nucl. Fusion,

43(2003)1197.

[28] KESNER, J., RAMOS, J.J., and

LUCKHARDT, S.C., Nucl. Fusion, 34(1994)795.
[29] JARDIN, S.C. and SCHMIDT, J.A., Nucl. Fusion 38(1998)1105.

[30] KUGEL, H., et al., Proc. of 17th IEEE/NPSS Symposium on Fusion Engineering, 1997, San Diego.

[31] STANGEBY, P.C., Nucl. Fusion, 43(2003)L1-L5.

[32] CHUDNOVSKIY, A.N., et al., Nucl. Fusion 43(2003)681.

[33] LUXON, J.L and DAVIS, L.G., Fusion

Technol. 8(1985)441.

[34] TURNBULL, A.D., et al., Nucl. Fusion

42(2002)917.

[35] STRAIT, E.J., Rev. Sci. Instrum.

67(1996) 2538

[36] AUSTIN, M.E., J. LOHR, Rev. Sci. Instrum., Vol. 74, p.1457 (2003).

[37] CARLSTROM, T.N., et al., Rev. Sci. Instrum. 63(1992)4901.

[38] COLCHIN, R.J., et al., "The Filterscope,"

General Atomics DIII-D Physics Memo 0007

(2001).

[39] GOHIL, P., et al., Proc. Of 14th Symp. on

Fusion Engineering, San Diego, CA, 1992, Vol. 2

(IEEE, New York, 1993), p. 1199.

[40] SNIDERR.T., EVANKO R. and HASKOVEC

J., Rev. Sci. Instrum. 59(1988)1807

[41] LAO, L.L., et al., Nucl. Fusion,

25(1985)1161.

[42] OKABAYASHI, M., et al., Plasma Phys.

Control. Fusion, 44(2002)B339

[43] BURRELL, K.H., et al., Phys. Plasmas,

8(2001)2153.

[44] TAKAHASHI, H., et al., Nucl. Fusion

42(2002)442

[45] BIALEK, J., "Time Domain VALEN

Calculations," Workshop on Active Control of

MHD Stability, AUSTIN, Texas, Nov. 3-5, 2003.

[46] TAKAHASHI, H., et al., "Scrape-off Layer

Current (SOLC) as a Dynamic Source of Error

Field," Error Magnetic Field Workshop,

Albuquerque, NM, Oct. 31-Nov. 1, 2003.

[47] FOREST, C., "Stabilization of the Resistive

Wall Mode Using Moving Metal Walls,"

Workshop on Active Control of MHD Stability,

AUSTIN, Texas, Nov. 3-5, 2003. 


\section{External Distribution}

Plasma Research Laboratory, Australian National University, Australia

Professor I.R. Jones, Flinders University, Australia

Professor João Canalle, Instituto de Fisica DEQ/IF - UERJ, Brazil

Mr. Gerson O. Ludwig, Instituto Nacional de Pesquisas, Brazil

Dr. P.H. Sakanaka, Instituto Fisica, Brazil

The Librarian, Culham Laboratory, England

Mrs. S.A. Hutchinson, JET Library, England

Professor M.N. Bussac, Ecole Polytechnique, France

Librarian, Max-Planck-Institut für Plasmaphysik, Germany

Jolan Moldvai, Reports Library, Hungarian Academy of Sciences, Central Research Institute for Physics, Hungary

Dr. P. Kaw, Institute for Plasma Research, India

Ms. P.J. Pathak, Librarian, Institute for Plasma Research, India

Ms. Clelia De Palo, Associazione EURATOM-ENEA, Italy

Dr. G. Grosso, Instituto di Fisica del Plasma, Italy

Librarian, Naka Fusion Research Establishment, JAERI, Japan

Library, Laboratory for Complex Energy Processes, Institute for Advanced Study, Kyoto University, Japan

Research Information Center, National Institute for Fusion Science, Japan

Dr. O. Mitarai, Kyushu Tokai University, Japan

Dr. Jiangang Li, Institute of Plasma Physics, Chinese Academy of Sciences, People's Republic of China

Professor Yuping Huo, School of Physical Science and Technology, People's Republic of China

Library, Academia Sinica, Institute of Plasma Physics, People's Republic of China

Librarian, Institute of Physics, Chinese Academy of Sciences, People's Republic of China

Dr. S. Mirnov, TRINITI, Troitsk, Russian Federation, Russia

Dr. V.S. Strelkov, Kurchatov Institute, Russian Federation, Russia

Professor Peter Lukac, Katedra Fyziky Plazmy MFF UK, Mlynska dolina F-2, Komenskeho Univerzita, SK-842 15 Bratislava, Slovakia

Dr. G.S. Lee, Korea Basic Science Institute, South Korea

Institute for Plasma Research, University of Maryland, USA

Librarian, Fusion Energy Division, Oak Ridge National Laboratory, USA

Librarian, Institute of Fusion Studies, University of Texas, USA

Librarian, Magnetic Fusion Program, Lawrence Livermore National Laboratory, USA

Library, General Atomics, USA

Plasma Physics Group, Fusion Energy Research Program, University of California at San Diego, USA

Plasma Physics Library, Columbia University, USA

Alkesh Punjabi, Center for Fusion Research and Training, Hampton University, USA

Dr. W.M. Stacey, Fusion Research Center, Georgia Institute of Technology, USA

Dr. John Willis, U.S. Department of Energy, Office of Fusion Energy Sciences, USA

Mr. Paul H. Wright, Indianapolis, Indiana, USA 
The Princeton Plasma Physics Laboratory is operated by Princeton University under contract with the U.S. Department of Energy.

\author{
Information Services \\ Princeton Plasma Physics Laboratory \\ P.O. Box 451 \\ Princeton, NJ 08543
}

Phone: 609-243-2750

Fax: 609-243-2751

e-mail: pppl_info@pppl.gov

Internet Address: http://www.pppl.gov 